\title{
Warping the Kähler potential of F-theory/IIB flux compactifications
}

\author{
Luca Martucci \\ Dipartimento di Fisica ed Astronomia "Galileo Galilei", Università di Padova, \\ and INFN — Sezione di Padova, \\ Via Marzolo 8, 35131 Padova, Italy \\ E-mail: luca.martucci@pd.infn.it
}

ABSTRACT: We identify the low-energy Kähler potential of warped F-theory/IIB flux compactifications whose light spectrum includes universal, Kähler, axionic and mobile D3-brane moduli. The derivation is based on four-dimensional local superconformal symmetry and holomorphy of brane instanton contributions and it reproduces and generalises previous partial results. We compute the resulting kinetic terms, which show their explicit dependence on the warping. The Kähler potential satisfies the no-scale condition and produces, at leading order in the large volume expansion, a specific correction to the unwarped Kähler potential.

Keywords: Flux compactifications, F-Theory, Supersymmetric Effective Theories

ARXIV EPRINT: 1411.2623 


\section{Contents}

1 Introduction and summary 1

2 Background structure $\quad 3$

3 Kähler potential from warped volume $\quad 4$

3.1 The conformal Kähler potential 5

3.2 The conformal compensator and the Kähler potential 6

4 Universal modulus and Kähler potential $\quad 8$

5 Decoding the Kähler potential $\quad 9$

$\begin{array}{ll}5.1 \text { Decoding the moduli dependence } & 12\end{array}$

$\begin{array}{ll}5.2 \text { Chiral fields and Kähler potential } & 14\end{array}$

5.3 A comment on D3-brane instantons 16

$\begin{array}{ll}5.4 \text { The } h^{1,1}=1 \text { case } & 17\end{array}$

$6 \quad$ Warped kinetic terms and no-scale condition $\quad 17$

$\begin{array}{lll}7 & \text { Large moduli limit } & 20\end{array}$

$\begin{array}{lll}8 & \text { Including axions } & 21\end{array}$

8.1 Closed string axionic chiral fields 22

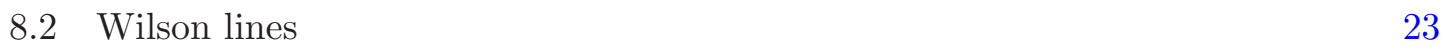

8.3 Complete Kähler potential for $h_{+}^{1,1}=1 \quad 23$

8.4 Kähler metric and no-scale structure 24

$\begin{array}{ll}\text { A Derivatives of geometric moduli } & \mathbf{2 5}\end{array}$

$\begin{array}{lll}\text { A.1 Useful preliminary formulas } & 25\end{array}$

$\begin{array}{lll}\text { A.2 Derivatives in absence of axionic chiral fields } & 26\end{array}$

$\begin{array}{lll}\text { A.3 Derivatives in presence of axionic chiral fields } & 27\end{array}$

$\begin{array}{lr}\text { B A simple example } & 27\end{array}$

\section{Introduction and summary}

Warped F-theory/IIB flux compactifications [1-7] play a prominent role in several phenomenologically inspired string constructions, for reviews see for instance [8, 9]. In particular, the flux allows to stabilise several moduli at tree-level and at the same time back-reacts on the geometry by generating a non-trivial warping, which can lead to interesting physical effects, as discussed e.g. in [5, 10-18]. 
However, a systematic understanding of the low-energy effective theory of such flux compactifications is, so far, still missing. The most common approach is to use the effective theory obtained from direct dimensional reduction by neglecting the back-reaction of fluxes and other sources, approximating the warping to a constant [19-23]. On the other hand, attempts to repeat a similar direct dimensional reduction by including a non-trivial warping, see for instance [10, 13, 24-29], are technically quite involved. These complications obscure the $N=1$ supersymmetric structure, which should eventually show up in the effective theory. Indeed, conclusive general answers have been so far reached only in few particular sub-cases.

In this paper, following [30,31], we use an alternative strategy to identify the effective Kähler potential of warped F-theory/IIB compactifications. This strategy is based on the interplay between ten-dimensional geometry, four-dimensional local superconformal symmetry and holomorphy of brane instantons. In section 2 we review the structure of the warped F-theory/IIB backgrounds and in section 3 we adapt the strategy of $[30,31]$ to them. As it happens in the constant warping approximation, in general the Kähler potential can only be defined implicitly. We omit from our discussion the moduli associated with the axio-dilaton, the complex structure and the position of seven-branes, which typically get a mass from the flux-induced tree-level superpotential. Hence, in section 4, we argue that the implicit Kähler potential has the very simple form

$$
K=-3 \log \hat{a}+\text { const }
$$

where $\hat{a}$ is a specific parametrisation of the universal modulus. The formula (1.1) is the warped counterpart of the analogous implicit formula $K_{\text {unwarped }} \simeq-2 \log V$, where $V$ is the internal volume, obtained in the constant warping approximation [19-23].

In order to make (1.1) explicit, one should know how $\hat{a}$ depends on the chiral fields $\varphi^{A}$ which properly parametrise the scalar sector of the $N=1$ four-dimensional effective theory. This issue is addressed in section 5, focusing on the case in which only the universal, Kähler and mobile D3-brane moduli are present. By using probe supersymmetric brane instantons, we introduce a natural set of chiral fields $\varphi^{A}$ and identify the relevant dependence thereof on the background moduli. This implicitly defines $\hat{a}=\hat{a}(\varphi, \bar{\varphi})$ and then, from (1.1), $K=K(\varphi, \bar{\varphi})$.

As we discuss in section 6, even though the Kähler potential (1.1) is defined only implicitly, it is actually possible to explicitly compute the associated low-energy kinetic terms. Furthermore, we show that the Kähler potential (1.1) is of no-scale type [32, 33]. This result is actually required by the physical consistency of the theory and then provides a strong consistency check.

Our results exhibit the precise contribution of mobile D3-branes, background fluxes and the remaining D3-charge sources to the effective Kähler potential. The contribution of D3branes had been already considered before under certain restrictions or approximations [10, $13,19,27,34]$. On the other hand, to best of our knowledge, the contribution of fluxes is completely new. As discussed in section 7 , by considering the large universal modulus limit, one can make more manifest the warping-induced correction to the Kähler potential with respect to the unwarped case. In particular, we explicitly identify the leading correction 
as a function of the Kähler moduli, the D3-brane positions, the fluxes and the other D3charge sources.

In section 8 , going to a weakly-coupled regime for technical simplicity, we show how one can adopt the same approach to incorporate the chiral fields associated with purely axionic moduli, neglected so far. All the steps work similarly as in the previous sections. In particular, one can compute the explicit form of the kinetic terms and verify the noscale condition.

Finally, appendix A contains some technical details and appendix B describes the application of the general results of the paper to a simple concrete class of $N=1$ flux compactifications on $T^{6} / \mathbb{Z}_{2}$ [35], for which the Kähler potential can be made fully explicit.

In this paper we do not explicitly consider the incorporation of other sectors of the effective theory, as for instance the gauge sector and the chiral matter, and the implications of higher-order corrections to the leading ten-dimensional supergravity theory. The investigation of these aspects is left to the future.

\section{$2 \quad$ Background structure}

Let us review the structure of warped F-theory/IIB compactifications, by using the type IIB description of these vacua [3-6]. These vacua have an Einstein frame metric of the form

$$
\mathrm{d} s_{10}^{2}=e^{2 D} \mathrm{~d} s_{\mathbb{R}^{1,3}}^{2}+e^{-2 D} \mathrm{~d} s_{X}^{2}
$$

where $\mathrm{d} s_{\mathbb{R}^{1,3}}^{2}$ is the flat Minkowski four-dimensional metric, the internal space $X$ is Kähler and the warping $e^{2 D}$ is generically non-constant along the internal directions. Furthermore for F-theory backgrounds, namely in presence of 7-branes, the axio-dilaton $\tau \equiv C_{0}+\mathrm{i} e^{-\phi}$ varies holomorphically along $X$ and is allowed to undergo non-trivial $\mathrm{SL}(2, \mathbb{Z})$ monodromies. The Ricci tensor $R^{X}$ associated with the internal Kähler metric $\mathrm{d} s_{X}^{2}$ is related to the dilaton $\phi$ by the formula:

$$
R_{i \bar{\jmath}}^{X}=\partial_{i} \partial_{\bar{\jmath} \phi}
$$

In addition, there can be a non-trivial three-form flux $G_{3}=F_{3}-\mathrm{i} e^{-\phi} H_{3}$, with $F_{3}=$ $\mathrm{d} C_{2}-C_{0} H_{3}$, which must be imaginary-self-dual (ISD) on $X, *_{X} G_{3}=\mathrm{i} G_{3}$. In other words $G_{3}$ can have non-vanishing primitive $(2,1)$ or $(0,3)$ components. Supersymmetry, which we will mostly assume, requires the $(0,3)$ component to be absent. In addition there can be supersymmetric world-volume fluxes supported on the world-volume of the seven branes. Furthermore, the R-R $F_{5}$ is also non-trivial and its form is directly linked to the warping by

$$
F_{5}=*_{X} \mathrm{~d} e^{-4 D}+\mathrm{d} x^{0123} \wedge \mathrm{d} e^{4 D}
$$

where $*_{X}$ is computed by using the Kähler metric $\mathrm{d} s_{X}^{2}$.

The $F_{5}$ Bianchi identity is

$$
\mathrm{d} F_{5}-H_{3} \wedge F_{3}=-Q_{6}^{\text {loc }}
$$

where $Q_{6}^{\text {loc }}$ denotes the localised D3-charge current

$$
Q_{6}^{\mathrm{loc}}=\ell_{\mathrm{s}}^{4} \sum_{I \in \mathrm{D} 3 \text { 's }} \delta_{I}^{6}-\frac{1}{4} \ell_{\mathrm{s}}^{4} \sum_{O \in \mathrm{O} 3 \text { 's }} \delta_{O}^{6}+\ldots
$$


We have introduced the string length $\ell_{\mathrm{s}} \equiv 2 \pi \sqrt{\alpha^{\prime}}$ and the six-form currents $\delta_{I}^{6}$ and $\delta_{O}^{6}$ localised at the position of the $I$-th D3-brane and of the $O$-th O3-plane respectively. ${ }^{1}$ In $Q_{6}^{\text {loc }}$ the ellipses denote other localised sources of D3-brane charge, induced by fluxes and curvature corrections on the 7-branes. By combining (2.3) and (2.4) one obtains the following equation for the warp factor:

$$
\Delta e^{-4 D}=\frac{1}{2 \operatorname{Im} \tau} G_{3} \cdot \bar{G}_{3}+*_{X} Q_{6}^{\mathrm{loc}}
$$

where we have introduced the Laplacian $\Delta \equiv-\nabla_{m} \nabla^{m}$.

The supersymmetric structure of these backgrounds is characterised by a Weyl Killing spinor $\epsilon$ of the form

$$
\epsilon=e^{\frac{D}{2}} \zeta_{\mathrm{R}} \otimes \eta
$$

where $\eta$ is an internal Weyl spinor satisfying the normalisation condition $\eta^{\dagger} \eta=1$, and $\zeta_{\mathrm{R}}$ is an external right-handed spinor. The spinor $\eta$ can be used to construct the holomorphic $(3,0)$-form $\Omega$ and the Kähler form $J$ as follows

$$
J_{m n}=-\mathrm{i} \eta^{\dagger} \gamma_{m n} \eta \quad \Omega_{m n p}=e^{\frac{\phi}{2}} \eta^{T} \gamma_{m n p} \eta
$$

These satisfy the normalisation condition

$$
\frac{1}{3 !} J \wedge J \wedge J=\frac{\mathrm{i}}{8} e^{-\phi} \Omega \wedge \bar{\Omega}=\operatorname{dvol}_{X}
$$

where the volume form $\mathrm{dvol}_{X}$ is computed by using the internal Kähler metric $\mathrm{d} s_{X}^{2} \cdot{ }^{2}$

It is important to observe that this description of the background is intrinsically redundant. Indeed, one can always perform a constant rescaling of the four-dimensional metric

$$
\mathrm{d} s_{\mathbb{R}^{1,3}}^{2} \rightarrow e^{-2 \omega} \mathrm{d} s_{\mathbb{R}^{1,3}}^{2}
$$

if accompanied by $e^{2 D} \rightarrow e^{2 D+2 \omega}$ and $\mathrm{d} s_{X}^{2} \rightarrow e^{2 \omega} \mathrm{d} s_{X}^{2}$. Furthermore, in the spinorial ansatz (2.7) one can perform a constant phase change $\zeta_{\mathrm{R}} \rightarrow e^{\frac{i}{2} \alpha} \zeta_{\mathrm{R}}$, if accompanied by $\eta \rightarrow e^{-\frac{i}{2} \alpha} \eta$. Under such reparametrizations $\Omega$ transforms as

$$
\Omega \rightarrow e^{3 \omega-\mathrm{i} \alpha} \Omega
$$

while $J \rightarrow e^{2 \omega} J$.

\section{Kähler potential from warped volume}

In this section we discuss how the Kähler potential for warped F-theory/IIB compactifications is directly related to the warped volume of the internal space. The Kähler potential

\footnotetext{
${ }^{1}$ In general, the $p$-form current $\delta_{\Sigma}^{p}$ associated with a $6-p$ surface $\Sigma$ is such that, for any $(6-p)$-form $\alpha$ on $X, \int_{\Sigma} \alpha=\int_{X} \alpha \wedge \delta_{\Sigma}^{p}$.

${ }^{2}$ In presence of 7 -branes, $\Omega$ cannot be considered as a section of the canonical bundle $\mathcal{K}_{X}$. Rather, it is a section of $\mathcal{K}_{X} \otimes \mathcal{L}_{Q}$, where $\mathcal{L}_{Q}$ is a holomorphic line bundle associated with the non-trivial holomorphic $\tau$. Supersymmetry requires that $\mathcal{L}_{Q} \simeq \mathcal{K}_{X}^{-1}$ so that $\Omega$ is indeed globally defined and no-where vanishing.
} 
that we obtain has the same form of the Kähler potential proposed in [10, 13]. Our argument is based on local superconformal symmetry as in [30,31]. It is morally very close to arguments presented in other papers, as for instance in [23,36-39], in which the Kähler potential is also directly related to the volume-like pre-factor appearing in the dimensionally reduced Einsten-Hilbert term. On the other hand, the local superconformal symmetry provides a precise scheme in which this conclusion is reached in a neat and natural way.

\subsection{The conformal Kähler potential}

The backgrounds described in section 2 are characterised by several moduli, which in this section we collectively denote by $u^{a}$. In order to obtain the four-dimensional effective theory one should allow for various components of the ten-dimensional ansatz to be slowly varying functions of the external coordinates. In particular, the external flat metric $\mathrm{d} s_{\mathbb{R}^{1,3}}^{2}$ and the moduli must be promoted to dynamical fields $g_{\mu \nu}(x) \mathrm{d} x^{\mu} \mathrm{d} x^{\nu}$ and $u^{a}(x)$ respectively. Then, the vacuum metric ansatz (2.1) must be generalised to

$$
\mathrm{d} s_{10}^{2}=e^{2 D(y ; u)} g_{\mu \nu} \mathrm{d} x^{\mu} \mathrm{d} x^{\nu}+e^{-2 D(y ; u)} \mathrm{d} s_{X}^{2}(y ; u)+\ldots
$$

We have shown only the terms that correspond to the naive generalisation of (2.1). In fact, as discussed for instance in $[13,25,26,40]$, in presence of warping these two terms do not constitute a consistent KK ansatz by themselves, but must be supplemented by additional compensating terms (often referred to as 'compensators'), which depend on the derivatives of the space-time dependent moduli $\partial_{\mu} u^{a}(x)$. These terms should then fill in the ellipses appearing on the right-hand side of (3.1).

Now, in presence of warping, a standard dimensional reduction is generically quite cumbersome, in particular because of the compensating terms. However, since the vacua we start from are supersymmetric, one already knows that the four-dimensional theory must be supersymmetric. Furthermore, crucially, in the dynamical ansatz (3.1), the scaling symmetry (2.10) is promoted to a gauge symmetry under arbitrary Weyl transformations $g_{\mu \nu} \rightarrow e^{-2 \omega(x)} g_{\mu \nu}$. An analogous conclusion holds for the arbitrary phase transformation of the spinors entering the dynamical generalisation of (2.7). Hence, in particular (2.11) is generalised to

$$
\Omega \rightarrow e^{3 \omega(x)-\mathrm{i} \alpha(x)} \Omega
$$

We then see that the effective four-dimensional theory must be gauge invariant under such complexified Weyl transformation. This tells us that the effective theory can be naturally regarded as a superconformal supergravity. By definition, fields transforming by a phase $e^{w \omega(x)+\mathrm{i} c \alpha(x)}$ under the above Weyl-chiral symmetry are said to have Weyl-chiral weight $(w, c)$. Hence $\Omega$ has Weyl-chiral weight $(3,-1)$. We refer to [41] for an exhaustive discussion on $N=1$ superconformal supergravities.

One of the nice features of superconformal supergravity is that the Einstein-Hilbert term as well as the scalars' kinetic terms all derive from a D-term of the schematic form

$$
-3 \int \mathrm{d}^{4} \theta \mathcal{N}(\Phi, \bar{\Phi})
$$


Here $\Phi^{I}$ denote the conformal chiral multiplets and we refer to $\mathcal{N}(\Phi, \bar{\Phi})$ as the conformal Kähler potential. Consistency requires $\mathcal{N}(\Phi, \bar{\Phi})$ to have Weyl-chiral weight $(2,0)$.

A discussion of the precise meaning of (3.3) can be found in [41]. Here we just need few bosonic terms which appear once (3.3) is written in components:

$$
\frac{1}{2} \mathcal{N} R_{4}+3 \mathcal{N}_{I \bar{J}} \partial_{\mu} \Phi^{I} \partial^{\mu} \bar{\Phi}^{\bar{J}}+\ldots
$$

where $\mathcal{N}_{I \bar{J}}(\Phi, \bar{\Phi}) \equiv \partial_{I} \bar{\partial}_{\bar{J}} \mathcal{N}(\Phi, \bar{\Phi})$ and we use the same symbol for chiral multiplets as well as for their scalar components.

By appropriately performing the KK reduction one should be able to recover the terms appearing in (3.4). Of course, in order to reconstruct the scalars' kinetic terms one should properly take into account the compensating terms, which contain the derivatives of the moduli $\partial_{\mu} u^{a}$. However, there is a clear short-cut. The scalars' kinetic terms are defined by the conformal Kähler potential $\mathcal{N}(\Phi, \bar{\Phi})$, which also appears as the prefactor of the Einstein term in (3.4). Since such term does not contain space-time derivatives of the chiral fields, one can obtain $\mathcal{N}$ by plugging the naive ansatz (3.1) in the Einstein term of the ten-dimensional type IIB action

$$
\frac{2 \pi}{\ell_{\mathrm{S}}^{8}} \int \sqrt{-g_{10}} R_{10},
$$

simply ignoring the problematic compensating terms. In this way, one easily gets

$$
\mathcal{N}=\frac{4 \pi}{\ell_{\mathrm{s}}^{8}} \int_{X} e^{-4 D} \mathrm{dvol}_{X}
$$

Of course, this is not the end of the story, since (3.6) provides only a rather implicit formula for $\mathcal{N}(\Phi, \bar{\Phi}){ }^{3}$

\subsection{The conformal compensator and the Kähler potential}

Part of the possible background moduli correspond to the deformations of the axio-dilaton $\tau$, which encodes the value of the string coupling as well as the seven-brane positions, and of the holomorphic $(3,0)$ form $\Omega$, which specifies the complex structure of the Kähler space $X$. The dual M-theory perspective elegantly unify these data into the holomorphic $(4,0)$ form associated with the dual elliptically fibered Calabi-Yau four-fold. Although these moduli could in principle be included in the discussion, we assume them to be stuck at a given value. This condition can be indeed dynamically enforced by the presence of fluxes. This means that $\tau$ and $\Omega$ are fixed, up to the latter's overall normalisation, which cannot be fixed basically because of the gauge invariance (3.2). It is then convenient to isolate such degree of freedom by writing

$$
\Omega \equiv \ell_{\mathrm{s}}^{6} Y^{3} \Omega_{0}
$$

\footnotetext{
${ }^{3}$ In [30] a holomorphic parametrisation of the generic (massless and massive) fluctuations was introduced, which used the formalism of generalised complex geometry. By using this parametrisation, it was shown how the conformal Kähler potential (3.6) and an associated GVW-like superpotential [42] reproduce the complete set of ten-dimensional equations of general $N=1$ compactifications to $\mathrm{Mink}_{4}$ and $\mathrm{AdS}_{4}$ found in $[43]$.
} 
where $Y$ is a completely arbitrary complex parameter which transforms with Weyl-chiral weight $\left(1,-\frac{1}{3}\right)$

$$
Y \rightarrow e^{\omega(x)-\frac{i}{3} \alpha(x)} Y
$$

while $\Omega_{0}$ is some fiducial dimensionless holomorphic $(3,0)$ form, satisfying a normalisation condition

$$
\frac{\mathrm{i}}{8} \int_{X} e^{-\phi} \Omega_{0} \wedge \bar{\Omega}_{0}=\mathrm{v}_{0}
$$

with $\mathrm{v}_{0}$ being a dimensionless constant. ${ }^{4}$ For instance, one may set $\mathrm{v}_{0}=1$.

The complex parameter $Y$ appears as a chiral field in the low-energy superconformal effective theory and can be used as conformal compensator to gauge fix the theory to a standard Poincaré supergravity. A detailed description of the procedure can be found in [41] and we will be sketchy.

First, one can isolate the dependence of $\mathcal{N}$ on the conformal compensator by writing it as

$$
\mathcal{N}=|Y|^{2} e^{-\frac{1}{3} K(\varphi, \bar{\varphi})}
$$

where $\varphi^{A}$ denote chiral fields of Weyl-chiral weight $(0,0)$ parametrising the background moduli. Then, one can gauge-fix the superconformal symmetry by imposing

$$
Y=M_{\mathrm{P}} e^{\frac{1}{6} K(\varphi, \bar{\varphi})}
$$

This implies that $\mathcal{N} \equiv M_{\mathrm{P}}^{2}$ and the first term in (3.4) reduces to the standard Einstein term $\frac{1}{2} M_{\mathrm{P}}^{2} R_{4}$. On the other hand, after the gauge fixing (3.11) the kinetic terms appearing in (3.4) produce the more familiar kinetic terms

$$
-M_{\mathrm{P}}^{2} K_{A \bar{B}}(\varphi, \bar{\varphi}) \partial_{\mu} \varphi^{A} \partial^{\mu} \bar{\varphi}^{\bar{B}}
$$

where $K_{A \bar{B}} \equiv \partial_{A} \bar{\partial}_{\bar{B}} K$. Hence the function $K(\varphi, \bar{\varphi})$ introduced in (3.10) can be identified with the Kähler potential of standard $N=1$ supergravity.

In order to identify the ten-dimensional expression for $K$ we observe that the redefinition (3.7) is naturally associated with the redefinitions $e^{2 D} \equiv \ell_{\mathrm{S}}^{2}|Y|^{2} e^{2 A}, \mathrm{~d} s_{X}^{2} \equiv \ell_{\mathrm{S}}^{4}|Y|^{2} \mathrm{~d} s_{X, 0}^{2}$ and $J \equiv \ell_{\mathrm{s}}^{4}|Y|^{2} J_{0}$, so that the ten-dimensional metric now reads

$$
\mathrm{d} s_{10}^{2}=\ell_{\mathrm{S}}^{2} e^{2 A}|Y|^{2} \mathrm{~d} s_{4}^{2}+\ell_{\mathrm{s}}^{2} e^{-2 A} \mathrm{~d} s_{X, 0}^{2}
$$

where the metric $\mathrm{d} s_{X, 0}^{2}$ still satisfies (2.2). Notice that $\mathrm{dvol}_{X, 0} \equiv \frac{1}{3 !} J_{0} \wedge J_{0} \wedge J_{0}$ can be considered as a fiducial volume-form with normalisation fixed by (3.9):

$$
\int_{X} \operatorname{dvol}_{X, 0}=\frac{1}{3 !} \int_{X} J_{0} \wedge J_{0} \wedge J_{0}=\mathrm{v}_{0}
$$

In practice, this normalisation condition removes the overall rescaling from the set of possible deformations of the Kähler form $J_{0}$. Such rescaling parametrises the universal modulus in standard unwarped compactifications. On the other hand, as we will review in the next

\footnotetext{
${ }^{4}$ The factor $e^{-\phi}$ is necessary to make the integrand a proper volume-form even in presence of $\operatorname{SL}(2, \mathbb{Z})$ monodromies.
} 
section, in warped backgrounds the universal modulus is encoded in the warp factor and then no physical degree of freedom is lost in the normalisation condition (3.14).

We can now write the (implicit) form of the Kähler potential $K(\varphi, \bar{\varphi})$. From (3.10) and the above field redefinitions one obtains

$$
\begin{aligned}
K(\varphi, \bar{\varphi}) & =-3 \log \left(4 \pi \int_{X} e^{-4 A} \operatorname{dvol}_{X, 0}\right) \\
& \equiv-3 \log \left(\frac{1}{3 !} \int_{X} e^{-4 A} J_{0} \wedge J_{0} \wedge J_{0}\right)-3 \log 4 \pi
\end{aligned}
$$

This Kähler potential has the same form as the Kähler potential proposed in [10, 13].

\section{Universal modulus and Kähler potential}

In this section we show that the Kähler potential (3.15) admits a simple expression in terms of the universal modulus. So, we start by reviewing the origin of the universal modulus in warped F-theory/IIB compactifications. First notice that the rescaled warp-factor $e^{2 A}$ introduced in the previous section satisfies an equation formally identical to (2.6),

$$
\Delta_{0} e^{-4 A}=\frac{1}{\ell_{\mathrm{s}}^{4}} *_{0} Q_{6} \equiv \frac{1}{\ell_{\mathrm{s}}^{4}}\left(\frac{1}{2 \operatorname{Im} \tau} G_{3} \cdot \bar{G}_{3}+*_{0} Q_{6}^{\mathrm{loc}}\right)
$$

where all quantities are now computed by using the rescaled metric $\mathrm{d} s_{X, 0}^{2}$.

The universal modulus is readily identified by looking at (4.1) and noticing that it completely determines $e^{-4 A}$ up to an additive constant. One can identify the universal modulus $a$ with such a constant, by writing

$$
e^{-4 A}=a+e^{-4 A_{0}}
$$

where $e^{-4 A_{0}}$ is a particular solution of (4.1). Notice that the split (4.2) is not unique as one could shift $a \rightarrow a+c$ and $e^{-4 A_{0}} \rightarrow e^{-4 A_{0}}-c$. One can fix such redundancy by imposing

$$
\int_{X} e^{-4 A_{0}} \mathrm{dvol}_{X, 0}=\mathrm{v}_{0}^{\mathrm{w}}
$$

for an arbitrary constant $\mathrm{v}_{0}^{\mathrm{w}}$. Hence, recalling (3.14), we can write the Kähler potential (3.15) as follows

$$
K=-3 \log \left(\mathrm{v}_{0} a+\mathrm{v}_{0}^{\mathrm{w}}\right)-3 \log 4 \pi
$$

Consider now the particular solution $e^{-4 \hat{A}_{0}}$ of (4.1) provided by the Green's operator of the Laplacian $\Delta_{0}$. The associated Green's function $G\left(y ; y^{\prime}\right)$ satisfies the equation

$$
\Delta_{0, y} G\left(y ; y^{\prime}\right)=*_{0} \delta_{y}^{6}\left(y^{\prime}\right)-\frac{1}{\mathrm{v}_{0}}
$$

The particular solution $e^{-4 \hat{A}_{0}}$ is then given by

$$
e^{-4 \hat{A}_{0}(y)}=\frac{1}{\ell_{\mathrm{s}}^{4}} \int_{X} G\left(y ; y^{\prime}\right) Q_{6}\left(y^{\prime}\right)
$$


By definition, the Green's operator vanishes on constant functions and maps coexact functions to coexact functions, see for instance [44]. This implies that $e^{-4 \hat{A}_{0}(y)}$ defined by (4.6) is a coexact function on $X$, so that

$$
\hat{\mathrm{v}}_{0}^{\mathrm{w}} \equiv \int_{X} e^{-4 \hat{A}_{0}} \operatorname{dvol}_{X, 0}=\frac{1}{3 !} \int_{X} e^{-4 \hat{A}_{0}} J_{0} \wedge J_{0} \wedge J_{0} \equiv 0
$$

The particular solution (4.6) is associated with a particular parametrisation $\hat{a}$ of the universal modulus, defined by

$$
e^{-4 A}=\hat{a}+e^{-4 \hat{A}_{0}}
$$

By using this specific split of $e^{-4 A}$ and (4.7), the Kähler potential (3.15) takes the form

$$
K=-3 \log \left(\mathrm{v}_{0} \hat{a}\right)-3 \log 4 \pi
$$

We then see that the Kähler potential (3.15) reduces to a simple function of just the universal modulus $\hat{a}$ (or $a$ ). On the other hand, the formula (4.9) (or (4.4)) is still implicit. In order to make it explicit we need to understand how the universal modulus $\hat{a}$ (or $a$ ) is parametrised in terms of appropriate holomorphic coordinates $\varphi^{A}$. This issue will be addressed in the following sections.

Notice that we have been careful in not dropping out additional constants in $K$, which are irrelevant in the computation of the Kähler metric. Nevertheless, such terms could be relevant for the computation of other quantities, as the potential

$$
V_{4 \mathrm{D}}=\frac{1}{M_{\mathrm{P}}^{2}} e^{K}\left(K^{A \bar{B}} D_{A} W D_{\bar{B}} \bar{W}-3|W|^{2}\right)
$$

which depends on the normalisation of the superpotential $W$ as well. For instance, with our choice of the Kähler potential, the classical GVW superpotential [5, 42] has the following specific normalisation [30]

$$
W_{\text {tree }}=\frac{\pi M_{\mathrm{P}}^{3}}{\ell_{\mathrm{S}}^{2}} \int_{X} \Omega_{0} \wedge G_{3}
$$

For later convenience, let us recall that (4.11) depends just on the axio-dilaton, complexstructure moduli and seven-brane moduli, and is at the origin of their stabilisation, which we assume. Hence, in the present paper $W_{\text {tree }}$ can be considered as constant, $W_{\text {tree }} \equiv W_{0}$. For supersymmetric vacua, i.e. with $(2,1)$ and primitive $G_{3}, W_{0}=0$. On the other hand, as reviewed in section 2 , supersymmetry is broken if the ISD $G_{3}$ has a non-vanishing $(0,3)$ component, still preserving the ten-dimensional equations of motion. In this case $W_{0} \neq 0$.

\section{Decoding the Kähler potential}

In order to interpret (4.9), or (4.4), as a Kähler potential, it is necessary to identify an appropriate holomorphic parametrisation $\varphi^{A}$ of the background moduli. A key observation is that the background can be 'probed' by supersymmetric Euclidean D3-branes (or vertical M5-branes in the dual M-theory description). It is well known that these branes enter the path-integral as instantonic corrections and can generate new F-terms in the effective 
action. These terms contain a universal factor $e^{-S_{\mathrm{D} 3}}$, where $S_{\mathrm{D} 3}$ is the Euclidean onshell D3-brane action. Crucially, by four-dimensional supersymmetry, $S_{\mathrm{D} 3}$ must depend holomorphically on the chiral fields $\varphi^{A}$. ${ }^{5}$

Now, the bosonic Euclidean D3-brane action can be written as

$$
S_{\mathrm{D} 3}=S_{\mathrm{DBI}}-\mathrm{i} S_{\mathrm{CS}}
$$

where $S_{\mathrm{DBI}}$ and $S_{\mathrm{CS}}$ are the standard Dirac-Born-Infeld (DBI) and Chern-Simons (CS) terms. In fact, in presence of warping and fluxes, the definition of $S_{\mathrm{CS}}$ is problematic, since the R-R potentials are not globally defined, see for instance [45] for a discussion in the analogous case of heterotic compactifications.

On the other hand, $S_{\mathrm{DBI}}$ has no such ambiguities and one can in principle compute its dependence on the background moduli. At the same time $S_{\mathrm{DBI}}$ must be the real part of a chiral field. Hence, it can be used to identify the dependence of the real part of a set of chiral fields on the background moduli. As we will see, this is sufficient to implicitly define $\hat{a}$, and then the Kähler potential (4.9), as functions of chiral fields.

In this section we apply this strategy by assuming that no two-form axionic moduli and seven-brane Wilson lines are present. This condition is better expressed in the dual elliptically fibered Calabi-Yau four-fold $\hat{X}$, by requiring that $b^{3}(\hat{X})-b^{3}(X)=0$, see e.g. [23]. The inclusion of axions will be discussed in section 8 .

Hence, in addition to the universal modulus $\hat{a}$ discussed in section 4 , we allow for three other kinds of moduli. The $h^{1,1}(X)-1$ Kähler moduli describing the deformations of $J_{0}$ that preserve the normalisation condition $(3.14),{ }^{6}$ the $h^{2,2}(X)=h^{1,1}(X)$ moduli describing the $C_{4}$ axions, and the $6 \times N_{\mathrm{D} 3}$ (real) moduli describing the positions of the $N_{\mathrm{D} 3}$ D3-branes in the internal space. The latter have a natural holomorphic parametrisation provided by the bulk complex coordinates $z^{i}, i=1,2,3$. Hence, we denote the chiral fields describing the D3-brane positions by $Z_{I}^{i}$, with $I=1, \ldots, N_{\mathrm{D} 3}$. We then need to understand how the remaining moduli organise into chiral fields.

Let us introduce a set of integral closed two-forms $\omega^{a}$, whose associated cohomology classes $\left[\omega_{a}\right]$ provide a basis of $H^{2}(X ; \mathbb{Z})$, and expand the Kähler form $J_{0}$ in cohomology as follows

$$
\left[J_{0}\right]=v_{a}\left[\omega^{a}\right]
$$

Then the constraint (3.14) can be written as

$$
\frac{1}{3 !} v_{a} v_{b} v_{c} \mathcal{I}^{a b c}=\mathrm{v}_{0}
$$

where we have introduced the intersection matrix

$$
\mathcal{I}^{a b c} \equiv \int_{X} \omega^{a} \wedge \omega^{b} \wedge \omega^{c}
$$

\footnotetext{
${ }^{5}$ Notice that the following discussion can be straightforwardly applied to seven-brane four-dimensional gauge-couplings as well. Indeed, by dimensionally reducing the effective action of a D7-branes (or, by $\mathrm{SL}(2, \mathbb{Z})$ duality, of a more general seven-brane) wrapped on a divisor $D$, one readily gets the complexified gauge coupling $\tau_{\mathrm{YM}}=\frac{1}{2 \pi} S_{\mathrm{D} 3}$.

${ }^{6}$ We are implicitly assuming that the proper orientifold projections are satisfied and we are ignoring for simplicity the possible lift of Kähler moduli induced by flux primitivity conditions. These aspects can be easily taken into account, as in the concrete example on $T^{6} / \mathbb{Z}_{2}$ discussed in appendix B.
} 
The constraint (5.3) identifies $h^{1,1}-1$ Kähler moduli out of the $h^{1,1}$ parameters $v_{a}$. Hence we will refer to $v_{a}$ as constrained Kähler moduli.

Now, a supersymmetric D3-brane must wrap a homographic four-cycle (an effective divisor) $D$, with anti-self-dual world-volume flux $\mathcal{F}=\frac{1}{2 \pi} \ell_{\mathrm{s}}^{2} F_{\mathrm{D} 3}-\left.B_{2}\right|_{D}$. These conditions are equivalent to requiring that the D3-brane is calibrated in the generalised sense of [46], so that the associated on-shell DBI action reduces to ${ }^{7}$

$$
S_{\mathrm{DBI}}=\pi \int_{D} e^{-4 A} J_{0} \wedge J_{0}-\frac{\pi}{\ell_{\mathrm{s}}^{4}} \int_{D} e^{-\phi} \mathcal{F} \wedge \mathcal{F}
$$

Notice that the second term does not depend on the background moduli we are considering in this section. So, for the moment, it is a constant and we can ignore it.

Now, there are $h^{1,1}(X)$ independent divisors $D^{a}$ and we choose the $\left[\omega^{a}\right] \in H^{2}(X ; \mathbb{Z})$ to be Poincaré dual to such divisors:

$$
\left[\omega^{a}\right]=\mathrm{PD}_{X}\left(D^{a}\right)
$$

By wrapping D3-branes along linear combinations of the divisors $D^{a}$, the associated $S_{\mathrm{DBI}}$ 's can detect all geometric moduli, i.e. universal modulus as well as all $h^{1,1}(X)-1$ constrained Kähler moduli $v_{a}$. On the other hand $S_{\mathrm{CS}}$ would detect the $h^{1,1}(X) \mathrm{R}-\mathrm{R}$ axions, which should complexify the geometric moduli. However, since the Kähler potential (4.9) (or (4.4)) depends just on geometric moduli, we do not actually need the information captured by $S_{\mathrm{CS}}$.

We can then focus on the integrals

$$
I^{a} \equiv \frac{1}{2} \int_{D^{a}} e^{-4 A} J_{0} \wedge J_{0}
$$

According to the arguments presented above, these must correspond to $h^{1,1}(X)$ chiral fields $\rho^{a}$ through a relation of the form

$$
I^{a}=\operatorname{Re} \rho^{a}+(\mathrm{hol}+\overline{\mathrm{hol}})
$$

where the $(\mathrm{hol}+\overline{\mathrm{hol}})$ contribution is the real part of some holomorphic quantity depending only on the remaining chiral fields.

Of course, deciding what to include in $(\mathrm{hol}+\overline{\mathrm{hol}})$ and what to absorb in the definition of $\operatorname{Re} \rho^{a}$ is a matter of choice. Indeed, possible different choices are related by a redefinition of the form $\rho^{a} \rightarrow \rho^{a}+$ hol, which preserves the holomorphic parametrisation of the chiral fields. In the following, our choice will be the minimal one. Namely, whenever we will be able to isolate a contribution to $I^{a}$ of the form hol $+\overline{\text { hol }}$ (not depending on $\hat{a}$ and $v_{a}$ ) we will omit it from the definition of $\operatorname{Re} \rho^{a}$.

\footnotetext{
${ }^{7}$ See [47-49] for more details on the supersymmetric structure of D3-brane instantons in F-theory compactifications, in the IIB framework adopted in the present paper.
} 


\subsection{Decoding the moduli dependence}

In order to proceed, we need to decode the dependence of the integrals (5.7) on the background moduli. Let us first use the decomposition (4.8) and write $I^{a}$ as

$$
I^{a} \equiv \hat{a} \mathcal{V}^{a}(v)+\frac{1}{2} \int_{D^{a}} e^{-4 \hat{A}_{0}} J_{0} \wedge J_{0}
$$

where

$$
\mathcal{V}^{a}(v) \equiv \frac{1}{2} \int_{D^{a}} J_{0} \wedge J_{0}=\frac{1}{2} \mathcal{I}^{a b c} v_{b} v_{c}
$$

The effect of warping is encoded in the second term on the r.h.s. of (5.9), which can depend on the constrained Kähler moduli $v_{a}$ and on the D3-brane moduli $Z_{I}^{i}$. In order to learn more about such dependence let us write the second term on the r.h.s. of (5.9) as

$$
\left.\frac{1}{2} \int_{X} e^{-4 \hat{A}_{0}} J_{0} \wedge J_{0} \wedge \delta_{D^{a}}^{2}=\int_{X} e^{-4 \hat{A}_{0}}\left(J_{0}\right\lrcorner \delta_{D^{a}}^{2}\right) \mathrm{dvol}_{X, 0}
$$

where $\delta_{D^{a}}^{2}$ is the two-form current (cf. footnote 1) associated with the divisor $D^{a}$ and

$$
\left.J_{0}\right\lrcorner \delta_{D^{a}}^{2} \equiv \frac{1}{2} J_{0}^{m n}\left(\delta_{D^{a}}^{2}\right)_{m n}
$$

Now, the localised term $\left.J_{0}\right\lrcorner \delta_{D^{a}}^{2}$ admits a nice explicit realisation in terms of the section $\zeta^{a}(z)$ of the line bundle $\mathcal{O}_{X}\left(D^{a}\right)$ whose vanishing locus identifies $D^{a}: D^{a}=\left\{\zeta^{a}=0\right\}$. Indeed, the Poincaré-Lelong equation

$$
\mathrm{i} \partial \bar{\partial} \operatorname{Re}\left(\log \zeta^{a}\right)=\pi \delta_{D^{a}}^{2}
$$

implies that

$$
\left.J_{0}\right\lrcorner \delta_{D^{a}}^{2}=-\frac{1}{2 \pi} \Delta_{0} \operatorname{Re} \log \zeta^{a}(z)
$$

Here we have used the fact that, since the internal space is Kähler, the Laplacian can be written as

$$
\left.\Delta_{0}=-2 g^{i \bar{\jmath}} \partial_{i} \bar{\partial}_{\bar{\jmath}}=-2 J_{0}\right\lrcorner(\mathrm{i} \partial \bar{\partial})
$$

By plugging (5.14) into (5.11), one could be tempted to integrate by parts $\Delta_{0}$ and then use (4.1). However, such procedure would not be admissible, since $\zeta^{a}(z)$ is a section of a non-trivial line bundle and then one would end with a meaningless expression.

In order to find a way out of this problem, let us choose the integral two-forms $\omega^{a}$ to be $(1,1)$ and harmonic, which is always possible within their cohomology class. Since $J_{0}$ is harmonic, we can promote the cohomological identity (5.2) to the pointwise identity

$$
J_{0}=v_{a} \omega^{a}
$$

It is important to realise that, in general, the $\omega^{a}$ 's actually depend on the constrained Kähler moduli $v_{a}$.

Now, by the $\partial \bar{\partial}$-lemma we can introduce a set of $h^{1,1}(X)$ local 'potentials' $\kappa^{a}(z, \bar{z} ; v)$, which generically depend also on the $v_{a}$ 's, such that

$$
\omega^{a}=\mathrm{i} \partial \bar{\partial} \kappa^{a}
$$


Clearly the $\kappa^{a}$ 's cannot be extended to global functions, since the $\omega^{a}$ 's are non-trivial in cohomology. Indeed, $e^{-2 \pi \kappa^{a}}$ can be identified with a metric on the line bundle $\mathcal{O}_{X}\left(D^{a}\right)$ associated with the divisor $D^{a}$, whose curvature is given by $2 \pi \omega^{a}$, see e.g. [50]. More explicitly, let us consider a holomorphic transformation

$$
\zeta^{a}(z) \rightarrow e^{2 \pi \chi^{a}(z)} \zeta^{a}(z)
$$

that relates the expressions of $\zeta^{a}(z)$ on different local patches. Then, $\kappa^{a}(z, \bar{z} ; v)$ must correspondingly transform as

$$
\kappa^{a}(z, \bar{z} ; v) \rightarrow \kappa^{a}(z, \bar{z} ; v)+\chi^{a}(z)+\bar{\chi}^{a}(\bar{z})
$$

Hence, the combination

$$
\pi \kappa^{a}(z, \bar{z} ; v)-\operatorname{Re} \log \zeta^{a}(z) \equiv-\frac{1}{2} \log e^{-2 \pi \kappa^{a}}\left|\zeta^{a}\right|^{2}
$$

is a globally well defined function on $X$.

Notice now that, since we have chosen the closed integral two-forms $\omega^{a}$ to be harmonic, the functions $\left.J_{0}\right\lrcorner \omega^{a}$ are harmonic too, i.e. they are constant along $X$ (although in general they depend on the constrained Kähler moduli $v_{a}$ ). By setting $\left.d^{a}(v) \equiv J_{0}\right\lrcorner \omega^{a}$, we can rewrite (5.14) as

$$
\left.J_{0}\right\lrcorner \delta_{D^{a}}^{2}=\frac{1}{2 \pi} \Delta_{0}\left[\pi \kappa^{a}(z, \bar{z} ; v)-\operatorname{Re} \log \zeta^{a}(z)\right]+d^{a}(v)
$$

We see that we have obtained a formula for $\left.J_{0}\right\lrcorner \delta_{D^{a}}^{2}$ in terms of the globally well defined function (5.20). By plugging (5.21) into (5.11), the contribution containing the constant $d^{a}(v)$ vanishes because of (4.7), and we are left with

$$
\frac{1}{2 \pi} \int_{X} e^{-4 \hat{A}_{0}} \Delta_{0}\left(\pi \kappa^{a}-\operatorname{Re} \log \zeta^{a}\right) \operatorname{dvol}_{X, 0}
$$

Now we are legitimised to integrate by parts the Laplacian and, by using (4.1), we arrive at the following remarkable identity

$$
\frac{1}{2} \int_{D^{a}} e^{-4 \hat{A}_{0}} J_{0} \wedge J_{0}=\frac{1}{2 \pi \ell_{\mathrm{S}}^{4}} \int_{X}\left(\pi \kappa^{a}-\operatorname{Re} \log \zeta^{a}\right) Q_{6}
$$

We recall that the six-form $Q_{6}$ denotes the D3-brane charge density and must satisfy the tadpole condition

$$
\int_{X} Q_{6}=0
$$

Hence, even though both $\kappa^{a}$ and $\log \zeta^{a}$ are defined up to an additive constant, the identity (5.23) as well as all results that will follow from it are not affected by this ambiguity.

One can isolate the contribution of the mobile D3-branes to $Q_{6}$ by writing

$$
Q_{6} \equiv \ell_{\mathrm{s}}^{4} \sum_{I} \delta_{I}^{6}+Q_{6}^{\mathrm{bg}}
$$


where $Q_{6}^{\mathrm{bg}}$ is the D3-charge 6-form induced by bulk fluxes, O3-planes as well as other localised D3-charge sources:

$$
Q_{6}^{\mathrm{bg}}=F_{3} \wedge H_{3}-\frac{1}{4} \ell_{\mathrm{s}}^{4} \sum_{O \in \mathrm{O} 3} \delta_{\mathrm{s}}^{6}+\ldots
$$

By plugging (5.25) into (5.23) and inserting the result back in (5.9), we finally arrive at the following more explicit form for $I^{a}$ :

$$
I^{a}(v, Z, \bar{Z})=\hat{a} \mathcal{V}^{a}(v)+h^{a}(v)+\frac{1}{2} \sum_{I} \kappa^{a}\left(Z_{I}, \bar{Z}_{I} ; v\right)-\frac{1}{2 \pi} \operatorname{Re} \log \left[\prod_{I} \zeta^{a}\left(Z_{I}\right)\right]
$$

where $^{8}$

$$
h^{a}(v) \equiv \frac{1}{2 \pi \ell_{\mathrm{s}}^{4}} \int_{X}\left(\pi \kappa^{a}-\operatorname{Re} \log \zeta^{a}\right) Q_{6}^{\mathrm{bg}}
$$

We stress that $I^{a}$ depends on the specific choice of the divisor $D^{a}$, since $h^{a}(v)$ depends on the associated holomorphic section $\zeta^{a}(z)$. The choice of a different divisor $\tilde{D}^{a}$, homologous to $D^{a}$, would correspond to a different section $\tilde{\zeta}^{a}(z)$, and then to a different warping-induced contribution:

$$
\tilde{I}^{a}(v, Z, \bar{Z})=I^{a}(v, Z, \bar{Z})+\frac{1}{2 \pi \ell_{\mathrm{S}}^{4}} \int_{X} \operatorname{Re}\left(\log \zeta^{a}-\log \tilde{\zeta}^{a}\right) Q_{6}
$$

The second term on the r.h.s. contributes to the $(\mathrm{hol}+\overline{\mathrm{hol}})$ part in the prescription $(5.8)$ and can then be reabsorbed in a holomorphic redefinition of the chiral fields $\rho^{a}$.

\subsection{Chiral fields and Kähler potential}

We can now come back to the prescription (5.8) to express the set of chiral fields $\rho^{a}$, or more precisely their real part, in terms of the background moduli. Indeed, by (5.27) we are naturally led to the following identification

$$
\operatorname{Re} \rho^{a}=\hat{a} \mathcal{V}^{a}(v)+h^{a}(v)+\frac{1}{2} \sum_{I} \kappa^{a}\left(Z_{I}, \bar{Z}_{I} ; v\right)
$$

Notice that, by consistency, under the transformations (5.19) $\rho^{a}$ must transform as

$$
\rho^{a} \rightarrow \rho^{a}+\sum_{I} \chi^{a}\left(Z_{I}\right)
$$

Hence the chiral fields $\rho^{a}$ are non-trivially fibered over the moduli space $\mathcal{M}_{\mathrm{D} 3}$ of the D3branes ${ }^{9}$ which can be identified with $N_{\mathrm{D} 3}$ copies of the internal space $X$, modded out by

\footnotetext{
${ }^{8}$ Notice that $h^{a}(v)$ diverges to $-\infty$ if the divisor $D^{a}$ touches some O3-planes. This is a consequence of the localized negative tension associated with the O3-planes. This is expected to be an artefact of the effective supergravity description, which should be cured by some higher-order or non-perturbative physical effect, as it happens for O7-branes in F-theory. In any case, one may judiciously choose the divisors $D^{a}$, in order to avoid this issue.

${ }^{9}$ See for instance $[12]$.
} 
the permutation group $S_{N_{\mathrm{D} 3}}$. In other words, the total moduli space $\mathcal{M}_{\text {tot }}$ is given by a non-trivial fibration over $\mathcal{M}_{\mathrm{D} 3}$.

Now, the equations (5.30) implicitly define $\hat{a}$ and $v_{a}$ as functions of $\operatorname{Re} \rho^{a}, Z_{I}^{i}$ and $\bar{Z}_{I}^{\bar{i}}$. In particular, from (4.9), one can in principle write

$$
K(\rho, \bar{\rho}, Z, \bar{Z})=-3 \log \left[\mathrm{v}_{0} \hat{a}(\operatorname{Re} \rho, Z, \bar{Z})\right]+c_{0}
$$

where

$$
c_{0}=-3 \log 4 \pi
$$

Of course, the main difficulty is the identification of the explicit form of the function $\hat{a}(\operatorname{Re} \rho, Z, \bar{Z})$, for which unfortunately there is no general solution. Simple cases in which one can be more explicit are discussed in the following. Nevertheless, as we will see in section 6, one can compute the effective kinetic terms by using just (5.30) and (5.32).

In order to make the relation with the literature on unwarped compactifications more immediate, one can combine $\hat{a}$ and the constrained Kähler moduli $v_{a}$ into the following $h^{1,1}(X)$ unconstrained moduli

$$
\hat{v}_{a}=v_{a} \sqrt{a}
$$

These can be considered as moduli of an auxiliary Kähler form $\hat{J}=\hat{v}_{a} \omega^{a}=\sqrt{a} J_{0}$, which would have a real geometrical interpretation only if one could consistently neglect $e^{-4 \hat{A}_{0}}$ with respect to $\hat{a}$ in (4.8), i.e. only if the warping could be considered constant.

By using the moduli $\hat{v}_{a}$ the Kähler potential (5.32) can be written as

$$
K(\rho, \bar{\rho}, Z, \bar{Z})=-2 \log \left(\frac{1}{3 !} \mathcal{I}^{a b c} \hat{v}_{a} \hat{v}_{b} \hat{v}_{c}\right)-\log \mathrm{v}_{0}+c_{0}
$$

while the relations (5.30) become

$$
\operatorname{Re} \rho^{a}=\frac{1}{2} \mathcal{I}^{a b c} \hat{v}_{b} \hat{v}_{c}+h^{a}(\hat{v})+\frac{1}{2} \sum_{I} \kappa^{a}\left(Z_{I}, \bar{Z}_{I} ; \hat{v}\right)
$$

Here we have used the fact that we can write $\kappa^{a}(z, \bar{z} ; v)=\kappa^{a}(z, \bar{z} ; \hat{v})$ and $h^{a}(v)=h^{a}(\hat{v})$, since the harmonicity condition on $\omega^{a}$ does not change if it is defined by $\hat{J}$ instead of $J_{0}$.

The description of the Kähler potential provided by (5.35) and (5.36) is formally closer to the one obtained in $[19,20,23]$ in the constant warping approximation. Basically, the Kähler potential can be casted in the universal form (5.35), while the new ingredients like D3-branes and fluxes are completely encoded in the functional dependence $\hat{v}_{a}=$ $\hat{v}_{a}(\operatorname{Re} \rho, Z, \bar{Z})$ which should be obtained by inverting (5.36). In particular, in [19] an analogous contribution of the D3-branes was obtained by using the probe D3-brane effective action expanded around a given point. In our language, such contribution can be reproduced by expanding $Z_{I}^{i} \simeq Z_{(0) I}^{i}+\Phi_{I}^{i}$ and approximating $\kappa^{a}\left(Z_{I}, \bar{Z}_{I} ; \hat{v}\right) \simeq-\mathrm{i} \omega_{i \bar{\jmath}}\left(Z_{(0) I}, \bar{Z}_{(0) I}\right) \Phi_{I}^{i} \bar{\Phi}_{I}^{\bar{j}}$.

On the other hand, to best of our knowledge, the contribution of the background fluxes and of the other localized D3-charge sources to the definition of the chiral coordinates had never been explicitly considered so far. This is completely encoded in the functions $h^{a}(v) \equiv h^{a}(\hat{v})$. As we will see in section 6 , the presence of such terms is crucial in the derivation of the (warped) kinetic terms of the effective action. 
As a final comment, notice that the relations (5.30), or (5.36), involve the background complex structure and, more implicitly, the seven-brane positions, whose induced D3charge would contribute to $Q_{6}^{\mathrm{bg}}$. In this paper we are assuming the corresponding moduli to be frozen. However, in any more complete treatment that includes (part of) these moduli as dynamical, they would mix with the remaining moduli in a non-trivial way, producing a non-diagonal Kähler potential, see also [19, 30, 31].

\subsection{A comment on D3-brane instantons}

Consider a supersymmetric Euclidean D3-brane wrapping a divisor $D$, associated with a holomorphic section $\zeta_{D}(z)$. In homology, we can expand $D \simeq n_{a} D^{a}$. Proceeding as in subsection 5.1 and using (5.30), one arrives at the identity

$$
\frac{1}{2} \int_{D} e^{-4 A} J_{0} \wedge J_{0}=n_{a} \operatorname{Re} \rho^{a}-\frac{1}{2 \pi} \operatorname{Re} \sum_{I} \log \zeta_{D}\left(Z_{I}\right)+\frac{1}{2 \pi \ell_{\mathrm{S}}^{4}} \int_{X} \operatorname{Re}\left(n_{a} \log \zeta^{a}-\log \zeta_{D}\right) Q_{6}^{\mathrm{bg}}
$$

Hence, the factor $e^{-S_{\mathrm{D} 3}}$ appearing in the associated non-perturbative F-terms is proportional to

$$
\mathcal{A}^{\mathrm{bg}}\left[\prod_{I} \zeta_{D}\left(Z_{I}\right)\right] e^{-2 \pi n_{a} \rho^{a}}
$$

where we have introduced

$$
\mathcal{A}^{\mathrm{bg}}=\exp \left[\frac{1}{\ell_{\mathrm{s}}^{4}} \int_{X}\left(\log \zeta_{D}-n_{a} \log \zeta^{a}\right) Q_{6}^{\mathrm{bg}}\right]
$$

We have added an overall phase in order to complexify $\mathcal{A}^{\text {bg }}$ in a way naturally compatible with the bulk complex structure.

The combination (5.38) is well defined on the total moduli space $\mathcal{M}_{\text {tot }}$, since $\zeta_{D}(z)$ is a section of $\mathcal{O}_{X}(D) \simeq \mathcal{O}_{X}\left(n_{a} D^{a}\right)$, while from (5.31) it follows that $e^{-2 \pi n_{a} \rho^{a}}$ transforms as a section of $\mathcal{O}_{X}\left(-n_{a} D^{a}\right) \simeq \mathcal{O}_{X}(-D)$.

In particular, the presence of the factor $\prod_{I} \zeta_{D}\left(Z_{I}\right)$, which encodes the dependence on the D3-brane moduli, is in agreement with what was anticipated in [51] from an argument based on monodromy and holomorphy. Hence our procedure gives, as particular by-product, an explicit derivation of such prefactor which is in the same spirit, although more direct and general, as the derivation presented in [34] for certain conifold backgrounds, see also [52].

On the other hand, the factor $\mathcal{A}^{\text {bg }}$ contains the effect of the back-reaction of the other background sources, see (5.26). It does not depend on the moduli we consider in the present paper. However, as $\prod_{I} \zeta_{D}\left(Z_{I}\right)$, it may become relevant in more general fluxcompactifications that include more dynamical fields in the low-energy effective theory. ${ }^{10}$

\footnotetext{
${ }^{10}$ For instance, our results automatically incorporate the mechanism proposed in [53] to generate nonperturbatively induced Yukawa couplings.
} 


\subsection{The $h^{1,1}=1$ case}

Let us restrict to the particular case in which there are no constrained Kähler moduli $v_{a}$, i.e. $h^{1,1}(X)=1$, so that $H^{1,1}(X)$ is generated by the Kähler form $J_{0}$. It is then convenient to take it integrally quantised and choose as single integral two-form $\omega \equiv J_{0}$. This corresponds to setting the single constrained Kähler modulus $v \equiv 1$. Notice that in this case $h$ is just a constant. Hence it can be omitted from (5.30), which then reduces to

$$
\operatorname{Re} \rho=3 \mathrm{v}_{0} \hat{a}+\frac{1}{2} \sum_{I} k\left(Z_{I}, \bar{Z}_{I}\right)
$$

Here we have identified $\kappa(z, \bar{z})$ with the Kähler potential $k(z, \bar{z})$ associated with $J_{0}$, defined by

$$
\mathrm{i} \partial \bar{\partial} k=J_{0}
$$

Inverting (5.40) to write $\hat{a}=\hat{a}(\operatorname{Re} \rho, Z, \bar{Z})$, the Kähler potential (5.32) takes the form

$$
K=-3 \log \left[\operatorname{Re} \rho-\frac{1}{2} \sum_{I} k\left(Z_{I}, \bar{Z}_{I}\right)\right]+c_{0}+3 \log 3
$$

This is in agreement with the Kähler potential first proposed in [10] and further discussed in $[13,27,34]$. Notice that we could have started from (4.4) and used in (5.40) the more general universal modulus $a$ instead of $\hat{a}=a+\frac{\mathrm{v}_{0}^{\mathrm{w}}}{\mathrm{v}_{0}}$. This shift in the definition of $\operatorname{Re} \rho$ would then modify the functional dependence of $K$ as follows

$$
K=-3 \log \left[\operatorname{Re} \rho-\frac{1}{2} \sum_{I} k\left(Z_{I}, \bar{Z}_{I}\right)+3 \mathrm{v}_{0}^{\mathrm{w}}\right]+c_{0}+3 \log 3
$$

with $\mathrm{v}_{0}^{\mathrm{w}}$ as in (4.3), consistently with the results of $[26,27]$.

\section{Warped kinetic terms and no-scale condition}

In this section we derive the kinetic terms of the chiral fields $\rho^{a}$ and $Z_{I}^{i}$ defined by the Kähler potential (5.32). Let us again collectively denote these fields by $\varphi^{A}$. Then, according to (3.12) we have to compute the matrix $K_{A \bar{B}} \equiv \partial_{A} \partial_{\bar{B}} K$. Since $K$ is only implicitly defined, one needs to compute the derivatives of $a$ and $v_{a}$ with respect to $\rho^{a}$ and $Z_{I}^{i}$ by using (5.30). One of the main difficulties comes from the dependence of $\kappa^{a}(z, \bar{z} ; v)$ and $h(v)$ on the constrained Kähler moduli $v_{a}$. Nevertheless, one can actually compute these derivatives. This is discussed in detail in appendix A and the final results are in (A.18) and (A.21). Here we just mention that a key ingredient in the derivation is a non-trivial interplay, physically due to the tadpole condition (5.24), between the contributions of $\kappa^{a}\left(Z_{I}, \bar{Z}_{I} ; v\right)$ and $h(v)$.

As a preliminary step, we introduce the matrix

$$
M_{\mathrm{w}}^{a b} \equiv \int_{X} e^{-4 A} J_{0} \wedge \omega^{a} \wedge \omega^{b}
$$


Notice that $M_{\mathrm{w}}^{a b}$ cannot be written in terms of topological intersection numbers, because of the warping $e^{-4 A}$. Let us also define the warped metric

$$
\mathcal{G}_{a b}^{\mathrm{w}} \equiv \frac{1}{4 \mathrm{v}_{0} \hat{a}}\left[\frac{1}{2 \mathrm{v}_{0} \hat{a}} v_{a} v_{b}-\left(M_{\mathrm{w}}^{-1}\right)_{a b}\right]
$$

and

$$
\mathcal{A}_{i}^{a I}\left(Z_{I}, \bar{Z}_{I} ; v\right) \equiv \frac{\partial \kappa^{a}\left(Z_{I}, \bar{Z}_{I} ; v\right)}{\partial Z_{I}^{i}} \quad \overline{\mathcal{A}}_{\bar{\imath}}^{a I}\left(Z_{I}, \bar{Z}_{I} ; v\right) \equiv \frac{\partial \kappa^{a}\left(Z_{I}, \bar{Z}_{I} ; v\right)}{\partial \bar{Z}_{I}^{\bar{l}}}
$$

Notice that under the transformations (5.19), we have

$$
\begin{gathered}
\mathcal{A}_{i}^{a I}\left(Z_{I}, \bar{Z}_{I} ; v\right) \rightarrow \mathcal{A}_{i}^{a I}\left(Z_{I}, \bar{Z}_{I} ; v\right)+\frac{\partial \chi^{a}\left(Z_{I}\right)}{\partial Z_{I}^{i}} \\
\overline{\mathcal{A}}_{\bar{\imath}}^{a I}\left(Z_{I}, \bar{Z}_{I} ; v\right) \rightarrow \overline{\mathcal{A}}_{\bar{\imath}}^{a I}\left(Z_{I}, \bar{Z}_{I} ; v\right)+\frac{\partial \bar{\chi}^{a}\left(\bar{Z}_{I}\right)}{\partial \bar{Z}_{I}^{\bar{c}}}
\end{gathered}
$$

Now, by using (A.18) and (A.21) one can quite straightforwardly compute the Kähler metric $K_{A \bar{B}}$ associated with the Kähler potential (5.32). The result is

$$
K_{A \bar{B}} \equiv\left(\begin{array}{cc}
\frac{\partial K}{\partial \rho^{a} \partial \bar{\rho}^{b}} & \frac{\partial K}{\partial \rho^{a} \partial \bar{Z}_{J}^{j}} \\
\frac{\partial K}{\partial Z_{I}^{i} \partial \bar{\rho}^{b}} & \frac{\partial K}{\partial Z_{I}^{i} \partial \bar{Z}_{J}^{j}}
\end{array}\right)=\left(\begin{array}{cc}
\mathcal{G}_{a b}^{\mathrm{w}} & -\mathcal{G}_{a c}^{\mathrm{w}} \overline{\mathcal{A}}_{\bar{\jmath}}^{c J} \\
-\mathcal{A}_{i}^{c I} \mathcal{G}_{c b}^{\mathrm{w}} & \mathcal{G}_{c d}^{\mathrm{w}} \mathcal{A}_{i}^{c I} \overline{\mathcal{A}}_{\bar{\jmath}}^{d J}+\frac{1}{2 \mathrm{v}_{0} \hat{a}} \delta^{I J} g_{0, i \bar{\jmath}}\left(Z_{I}, \bar{Z}_{I}\right)
\end{array}\right)
$$

where the $g_{0, i \bar{\jmath}}(z, \bar{z})$ is the Kähler metric of the internal space $X$.

By using (6.5) we can write the kinetic terms (3.12) in the form

$$
\mathcal{L}_{\text {kin }}=-M_{\mathrm{P}}^{2} \mathcal{G}_{a b}^{\mathrm{w}} \nabla_{\mu} \rho^{a} \nabla^{\mu} \bar{\rho}^{b}-\frac{1}{2 \mathrm{v}_{0} \hat{a}} M_{\mathrm{P}}^{2} \sum_{I} g_{0, i \bar{\jmath}}\left(Z_{I}, \bar{Z}_{I}\right) \partial_{\mu} Z_{I}^{i} \partial^{\mu} \bar{Z}_{I}^{\bar{j}}
$$

where we have introduced the covariant derivatives

$$
\nabla_{\mu} \rho^{a} \equiv \partial_{\mu} \rho^{a}-\mathcal{A}_{i}^{a I} \partial_{\mu} Z_{I}^{i}
$$

and $\nabla_{\mu} \bar{\rho}^{a} \equiv\left(\nabla_{\mu} \rho^{a}\right)^{*}$, which are invariant under the transformations (5.31), taking (6.4) into account.

Notice that the second term on the r.h.s. of (6.6) is easily reproduced by probe D3branes. Indeed, focusing on a single D3-brane, from (4.9) and (3.11) it follows that $M_{\mathrm{P}}^{2}=$ $4 \pi \mathrm{v}_{0} \hat{a}|Y|^{2}$ so that the second term appearing in the r.h.s. of (6.6) can be written as

$$
-2 \pi|Y|^{2} g_{0, i \bar{\jmath}}(Z, \bar{Z} ; v) \partial_{\mu} Z^{i} \partial^{\mu} \bar{Z}^{\bar{\jmath}}
$$

which indeed coincides with the kinetic term obtained by expanding the DBI action of a probe D3-brane on the ten-dimensional metric (3.13). This provides a non-trivial check of our result.

Now, from (A.18) and (A.21) one can easily compute the first derivatives of the Kähler potential:

$$
K_{A}=\left(\frac{\partial K}{\partial \rho^{a}}, \frac{\partial K}{\partial Z_{I}^{i}}\right)=\left(-\frac{v_{a}}{2 v_{0} \hat{a}}, \frac{1}{2 \mathrm{v}_{0} \hat{a}} v_{a} \mathcal{A}_{i}^{a I}\right)
$$


and $K_{\bar{A}}=\left(K_{A}\right)^{*}$. Furthermore, the inverse of the Kähler metric (6.5) takes the form:

$$
K^{A \bar{B}}=\left(\begin{array}{cc}
\mathcal{G}_{\mathrm{w}}^{a b}+2 \mathrm{v}_{0} \hat{a} \sum_{L} g_{0}^{l \bar{m}}\left(Z_{L}, \bar{Z}_{L}\right) \mathcal{A}_{l}^{a L} \overline{\mathcal{A}}_{\bar{m}}^{b L} & 2 \mathrm{v}_{0} \hat{a} \mathcal{A}_{l}^{a L} g_{0}^{l \bar{\jmath}}\left(Z_{J}, \bar{Z}_{J}\right) \delta_{L J} \\
2 \mathrm{v}_{0} \hat{a} \delta_{I M} g_{0}^{i \bar{m}}\left(Z_{I}, \bar{Z}_{I}\right) \overline{\mathcal{A}}_{\bar{m}}^{b M} & 2 \mathrm{v}_{0} \hat{a} \delta_{I J} g_{0}^{i \bar{\jmath}}\left(Z_{I}, \bar{Z}_{I}\right)
\end{array}\right)
$$

where $g_{0}^{\bar{i} j}$ is the inverse of $g_{0, i \bar{\jmath}}$ and

$$
\mathcal{G}_{\mathrm{w}}^{a b} \equiv\left(M_{\mathrm{w}}^{a d} v_{d} v_{c}-4 \mathrm{v}_{0} \hat{a} \delta_{c}^{a}\right) M_{\mathrm{w}}^{c b}
$$

is the inverse of $\mathcal{G}_{a b}^{\mathrm{w}}$. Hence, from (6.9) and (6.10) one can straightforwardly check that

$$
K^{A \bar{B}} K_{A} K_{\bar{B}}=3
$$

That is, our warped Kähler potential is of no-scale type [32, 33].

In fact (6.12) had to be expected. Indeed supersymmetry can be broken, preserving the ten-dimensional equations of motion, if the classical superpotential (4.11) has a non-vanishing expectation value $W_{0} \neq 0$. In particular, these non-supersymmetric vacua still have a Minkowski external space. The no-scale condition (6.12) makes such tendimensional supersymmetry breaking mechanism naturally consistent with the effective four-dimensional supergravity viewpoint. Hence, (6.12) provides a further consistency check for our results.

So far we have used only chiral multiplets in the low-energy effective theory. On the other hand, one could dualise the chiral fields $\rho^{a}$ to linear multiplets. Let us denote the scalar components of the dual linear multiplets by $l_{a}$. They can be easily computed within the superconformal formalism, before imposing the gauge fixing condition (3.11) on the conformal compensator, and are given by $[54,55]$

$$
l_{a}=\frac{3}{4 \pi} \frac{\partial \mathcal{N}}{\partial \operatorname{Re} \rho^{a}}
$$

From (3.10) it follows that $\mathcal{N}=4 \pi|Y|^{2} \mathrm{v}_{0} \hat{a}$. Then, by using (A.18a) we obtain

$$
l_{a}=|Y|^{2} v_{a}
$$

That is, the scalars $l_{a}$ are just the components appearing in the expansion $J=\ell_{\mathrm{s}}^{2} l_{a} \omega^{a}$ of the non-normalised Kähler form $J \equiv \ell_{\mathrm{s}}^{2}|Y|^{2} J_{0}$ introduced in (2.8). This shows that the scalars $l_{a}$ have a more transparent geometrical interpretation, compared to the chiral fields $\rho^{a}$. The complexity of (5.30) could then be regarded as the result of performing the inverse duality from linear to chiral multiplets. See $[20,23]$ for a detailed discussion in the unwarped case and [31] for similar remarks in the case of generalised compactifications.

Finally, let us give a closer look at the warped matrix $M_{\mathrm{w}}^{a b}$ defined in (6.1), which encodes the non-trivial warping contribution to $\mathcal{G}_{a b}^{\mathrm{w}}$. In particular, by splitting $e^{-4 A}$ as in (4.8), $M_{\mathrm{w}}^{a b}$ can be rewritten as

$$
M_{\mathrm{w}}^{a b}=\hat{a} \mathcal{I}^{a b c} v_{c}+\int_{X} e^{-4 \hat{A}_{0}} J_{0} \wedge \omega^{a} \wedge \omega^{b}
$$


The second term on the r.h.s. of this equation is what makes $\mathcal{G}_{a b}^{\mathrm{w}}$ quantitatively different with respect to the corresponding metric in the constant-warping approximation [20,23].

Even though we generically expect the second term on the r.h.s. of (6.15) to be nontrivial, there are some particular cases in which it actually vanishes. Indeed, recall that $e^{-4 \hat{A}_{0}}$ satisfies the normalisation condition (4.7). Hence we see that the second term on the r.h.s. of (6.15) vanishes if

$$
J_{0} \wedge \omega^{a} \wedge \omega^{b}=C^{a b} J_{0} \wedge J_{0} \wedge J_{0}
$$

for some constants $C^{a b}$. The condition (6.16) is equivalent to requiring that the product of any harmonic $(1,1)$-form and any harmonic $(2,2)$-form gives a harmonic 6 -form. This is for instance the case for the simple class of $N=1$ compactifications on $X=T^{6} / \mathbb{Z}_{2}$ discussed in appendix B. More generically, such property is guaranteed if $X$ is geometrically formal [56] that is, if the product of any pair of harmonic forms gives a harmonic form. A similar conclusion was reached in [29] by studying the kinetic terms of the $C_{4}$ axionic moduli on warped Calabi-Yau three-folds.

\section{$7 \quad$ Large moduli limit}

It can be sensible to consider a regime in which $\operatorname{Re} \rho^{a}$ are all very large, $\operatorname{Re} \rho^{a} \gg 1$. This is indeed the regime in which the supergravity description of string theory is expected to be fully legitimate. Hence, it is natural to look for a perturbative solution of the inverted relations $\hat{a}=\hat{a}(\operatorname{Re} \rho, Z, \bar{Z})$ and $v_{a}=v_{a}(\operatorname{Re} \rho, Z, \bar{Z})$ in powers of $(\operatorname{Re} \rho)^{-1}$.

First expand $\hat{a}$ and $v_{a}$ as follows

$$
\hat{a}=\hat{a}^{(-1)}+\hat{a}^{(0)}+\mathcal{O}\left(\frac{1}{\operatorname{Re} \rho}\right) \quad v_{a}=v_{a}^{(0)}+v_{a}^{(1)}+\mathcal{O}\left(\frac{1}{(\operatorname{Re} \rho)^{2}}\right)
$$

Furthermore, by expanding the constraint (5.3) up to first order, one gets

$$
v_{a}^{(0)} v_{b}^{(0)} v_{c}^{(0)} \mathcal{I}^{a b c}=6 \mathrm{v}_{0} \quad v_{a}^{(0)} v_{b}^{(0)} v_{c}^{(1)} \mathcal{I}^{a b c}=0
$$

Hence the leading order contribution $\operatorname{Re} \rho^{a}=\frac{1}{2} \hat{a}^{(-1)} \mathcal{I}^{a b c} v_{b}^{(0)} v_{c}^{(0)}$ to (5.30) implicitly gives $\hat{a}^{(-1)}$ and $v_{a}^{(0)}$ in terms of $\operatorname{Re} \rho^{a}$. On the other hand, the next-to-leading order of (5.30) gives $a^{(0)}$ in terms of $v_{a}^{(0)}: 11$

$$
\hat{a}^{(0)}=-\frac{1}{3 \mathrm{v}_{0}} h\left(v^{(0)}\right)-\frac{1}{6 \mathrm{v}_{0}} \sum_{I} k\left(Z_{I}, \bar{Z}_{I} ; v^{(0)}\right)
$$

where $k \equiv v_{a} \kappa^{a}$ is nothing but the Kähler potential associated with $J_{0}$, as defined in (5.41), and we have introduced the quantities $M_{(0)}^{a b} \equiv \mathcal{I}^{a b c} v_{c}^{(0)}$ and $h\left(v^{(0)}\right) \equiv v_{a}^{(0)} h^{a}\left(v^{(0)}\right)$.

We can now consider $\hat{a}^{(-1)}$ and $v_{a}^{(0)}$ as the independent geometric moduli and suppress the superscripts for notational simplicity. Hence, by neglecting terms of order $(\operatorname{Re} \rho)^{-1}$ in the logarithm, the Kähler potential (5.32) can be approximated by

$$
K \simeq-3 \log \left[\mathrm{v}_{0} \hat{a}-\frac{1}{3} h(v)-\frac{1}{6} \sum_{I} k\left(Z_{I}, \bar{Z}_{I} ; v\right)\right]+c_{0}
$$

\footnotetext{
${ }^{11}$ One also obtains $v_{a}^{(1)}=\frac{1}{\hat{a}^{(-1)}}\left[\frac{1}{6 \mathrm{v}_{0}} v_{a}^{(0)} v_{b}^{(0)}-\left(M_{(0)}^{-1}\right) a b\right]\left[h^{b}\left(v^{(0)}\right)+\frac{1}{2} \sum_{I} \kappa^{b}\left(Z_{I}, \bar{Z}_{I} ; v^{(0)}\right)\right]$.
} 
where

$$
h(v) \equiv \frac{1}{2 \pi \ell_{\mathrm{s}}^{4}} \int_{X}\left[\pi k(z, \bar{z} ; v)-v_{a} \operatorname{Re} \log \zeta^{a}(z)\right] Q_{6}^{\mathrm{bg}}
$$

Alternatively, by using unconstrained Kähler parameters $\hat{v}_{a}=v_{a} \sqrt{\hat{a}}$ as in (5.34), associated with $\hat{J}=\hat{v}_{a} \omega^{a}$, the Kähler potential (7.4) can be written as

$$
K \simeq-2 \log \left[\frac{1}{3 !} \mathcal{I}^{a b c} \hat{v}_{a} \hat{v}_{b} \hat{v}_{c}-\frac{1}{2} h(\hat{v})-\frac{1}{4} \sum_{I} k\left(Z_{I}, \bar{Z}_{I} ; \hat{v}\right)\right]-\log \mathrm{v}_{0}+c_{0}
$$

In (7.4) and (7.6) the geometric moduli $\hat{a}$ and $v_{a}$, or $\hat{v}_{a}$, must be regarded as functions of $\operatorname{Re} \rho^{a}$ alone, implicitly defined by

$$
\operatorname{Re} \rho^{a}=\frac{1}{2} \hat{a} \mathcal{I}^{a b c} v_{b} v_{c} \equiv \frac{1}{2} \mathcal{I}^{a b c} \hat{v}_{b} \hat{v}_{c}
$$

In (7.4), or (7.6), the effect of D3-branes and fluxes appears directly in the Kähler potential, while the relation (7.7) between geometric moduli $\hat{v}_{a}$ and holomorphic moduli $\rho^{a}$ is formally the same as for constant warping compactifications [20, 23]. The modification due to D3-branes is of the same form proposed in [10]. On the other hand, the effect of the fluxes and other D3-charge sources is encoded in $h(\hat{v})$. Notice that if we rescale $\hat{v}_{a} \rightarrow \lambda \hat{v}_{a}$, then $k(z, \bar{z} ; \hat{v})$ and $h(\hat{v})$ scale like homogeneous functions of degree one. Hence, by expanding the logarithm, we see that the overall correction to the Kähler potential scales with $\lambda^{-2}$. In other words, the first order correction scales like the $V^{-\frac{2}{3}}$, where $V \equiv \frac{1}{3 !} \mathcal{I}^{a b c} \hat{v}_{a} \hat{v}_{b} \hat{v}_{c}$ can be identified with the internal volume of the corresponding constant warping compactification. This scaling behaviour was also argued in [29] by studying the dimensionally reduced theory for axionic moduli.

\section{Including axions}

In the previous sections we assumed the absence of chiral fields corresponding to purely axionic moduli. In fact, they can be included by using the same strategy followed so far. The possible axionic chiral fields are more easily identified in the dual elliptically fibered Calabi-Yau four-fold $\hat{X}$, on which they are counted by $b^{3}(\hat{X})-b^{3}(X)$, see [23] for a detailed discussion. In such description we should use probe Euclidean M5-branes wrapping vertical divisors and, unfortunately, the M5-brane effective theory on non-trivial supergravity backgrounds is not very manageable [57, 58]. On the other hand, in the type IIB framework the axionic chiral fields are less easily identified, but one can use the Euclidean D3-brane action, which is under better control.

To avoid such technical complications and outline the general strategy to include axionic moduli, we will restrict to F-theory backgrounds admitting a weakly coupled description, with D7-branes and O7-planes and an almost constant axio-dilaton $\tau$. In this limit one can work in the Calabi-Yau double cover three-fold $\tilde{X}$ and distinguish between closed and open string axionic chiral fields. 


\subsection{Closed string axionic chiral fields}

Let us first focus on the closed string moduli. Introduce a set of harmonic $(1,1)$-forms $\chi_{\alpha}$ that are odd under the orientifold involution and form a basis for $H_{-}^{1,1}(\tilde{X})$. We can then fix a certain reference $B_{2}^{(0)}$ and $C_{2}^{(0)}$ satisfying the flux equations, and write $B_{2}=B_{2}^{(0)}+\Delta B_{2}$ and $C_{2}=C_{2}^{(0)}+\Delta C_{2}$, with $\Delta B_{2}$ and $\Delta C_{2}$ being closed harmonic $(1,1)$-forms. We can then expand $\Delta B_{2}$ and $\Delta C_{2}$ as follows

$$
\Delta B_{2}=\ell_{\mathrm{s}}^{2} b^{\alpha} \chi_{\alpha} \quad \Delta C_{2}=\ell_{\mathrm{s}}^{2} c^{\alpha} \chi_{\alpha}
$$

The moduli $b^{\alpha}$ and $c^{\alpha}$ are naturally combined into the following set of $h_{-}^{1,1}(\tilde{X})$ chiral fields:

$$
\beta^{\alpha}=c^{\alpha}-\tau b^{\alpha}
$$

In order to include them in the above discussion, we need to take into account the second term appearing in the on-shell supersymmetric DBI-action (5.5). By considering a Euclidean D3-brane wrapping an even divisor $D \subset \tilde{X}$ (that is, such that $\sigma(D)=D$ ), the second term on the r.h.s. of (5.5) can be written as

$$
-\frac{\pi}{2 \ell_{\mathrm{s}}^{4}} \int_{D} e^{-\phi} \mathcal{F}_{(0)} \wedge \mathcal{F}_{(0)}-\left(\frac{\pi}{\ell_{\mathrm{s}}^{2}} \int_{D} \mathcal{F}_{(0)} \wedge \chi_{\alpha}\right) \operatorname{Im} \beta^{\alpha}+\frac{\pi}{\operatorname{Im} \tau} \mathcal{M}_{\alpha \beta} \operatorname{Im} \beta^{\alpha} \operatorname{Im} \beta^{\beta}
$$

where $\mathcal{M}_{\alpha \beta} \equiv-\frac{1}{2} \int_{D} \chi_{\alpha} \wedge \chi_{\beta}$ and we have included an overall factor $\frac{1}{2}$ to take into account that we are working in the double cover $\tilde{X}$.

Taking (8.3) into account, the definition of $I^{a}$ given in (5.7) (written in the covering space) should be modified into

$$
I^{a}=\frac{1}{4} \int_{D} e^{-4 A} J_{0} \wedge J_{0}-\left(\frac{1}{2 \ell_{\mathrm{s}}^{2}} \int_{D} \mathcal{F}_{(0)} \wedge \chi_{\alpha}\right) \operatorname{Im} \beta^{\alpha}+\frac{1}{2 \operatorname{Im} \tau} \mathcal{M}_{\alpha \beta} \operatorname{Im} \beta^{\alpha} \operatorname{Im} \beta^{\beta}
$$

where we have omitted the contribution of the first term in (8.3), since moduli independent. The second term in (8.4) can be written as $(\mathrm{hol}+\overline{\mathrm{hol}})$. Hence, according to the general prescription (5.8), it can be ignored as well. Repeating the discussion which lead to (5.30), we then arrive at the identification

$$
\operatorname{Re} \rho^{a}=\hat{a} \mathcal{V}^{a}(v)+h^{a}(v)+\frac{1}{2} \sum_{I} \kappa^{a}\left(Z_{I}, \bar{Z}_{I} ; v\right)+\frac{1}{2 \operatorname{Im} \tau} \mathcal{M}_{\alpha \beta}^{a} \operatorname{Im} \beta^{\alpha} \operatorname{Im} \beta^{\beta}
$$

where

$$
\mathcal{M}_{\alpha \beta}^{a} \equiv-\frac{1}{2} \int_{\tilde{X}} \omega^{a} \wedge \chi_{\alpha} \wedge \chi_{\beta}
$$

As for the Kähler potential, it still takes the form (5.32), up to allowing $\hat{a}$ to depend also on $\operatorname{Im} \beta^{a}$. So (8.5) is the only modification we need to consider. In particular, the axionic contribution to (8.5) is identical to the corresponding contribution in the constant warping approximation [20]. 


\subsection{Wilson lines}

Finally, we incorporate the open string axions as well. Let us focus on a D7-brane wrapping an orientifold invariant divisor $\Sigma=\sigma(\Sigma)$. Then, the (orientifold odd) gauge field $A_{\mathrm{D} 7}$ supported on $\Sigma$ can be split into $A_{\mathrm{D} 7}^{(0)}+\Delta A_{\mathrm{D} 7}$, where $A_{\mathrm{D} 7}^{(0)}$ is some fixed background gauge field and $\Delta A_{\mathrm{D} 7}$ is an arbitrary harmonic one-form, which can be regarded as a Wilson line. The corresponding chiral fields $a^{\mathcal{I}}$ can be obtained by expanding the $(0,1)$ component $\Delta A_{\mathrm{D} 7}^{0,1}$ in a basis of harmonic $(0,1)$-forms $\eta_{\mathcal{I}} \in H^{0,1}(\Sigma)$ :

$$
\Delta A_{\mathrm{D} 7}^{0,1}=2 \pi a^{\mathcal{I}} \eta_{\mathcal{I}}
$$

Now, the gauge field $A_{\mathrm{D} 7}$ couples to a Euclidean D3-brane wrapping a divisor $D$ through its coupling with the chiral scalar $\beta$ supported on the intersection curve $\mathcal{C}=$ $\Sigma \cap D[49]$ :

$$
\frac{\mathrm{i}}{8 \pi} \int_{\mathcal{C}}\left[\partial \beta \wedge \bar{\partial} \beta-2 \partial \beta \wedge\left(A_{\mathrm{D} 3}^{0,1}-A_{\mathrm{D} 7}^{0,1}\right)+A_{\mathrm{D} 3}^{1,0} \wedge A_{\mathrm{D} 3}^{0,1}-2 A_{\mathrm{D} 3}^{1,0} \wedge A_{\mathrm{D} 7}^{0,1}+A_{\mathrm{D} 7}^{1,0} \wedge A_{\mathrm{D} 7}^{0,1}\right]
$$

This two-dimensional action contributes to $S_{\mathrm{D} 3}$. In order to identify the modification of (8.5) due to Wilson lines, we use the same strategy as above and we require $S_{\mathrm{D} 3}$ to depend homomorphically on the background chiral fields. The terms that do not depend on $A_{\mathrm{D} 7}$ can be ignored. Furthermore, the terms that are linear on $A_{\mathrm{D} 7}^{0,1}$ can be ignored as well, since they depend holomorphically on the Wilson lines $a^{I}$. On the other hand, the real quadratic term

$$
\frac{\mathrm{i}}{8 \pi} \int_{\mathcal{C}} A_{\mathrm{D} 7}^{1,0} \wedge A_{\mathrm{D} 7}^{0,1}
$$

must be taken into account. More specifically, by using the expansion (8.7), the definition of $\operatorname{Re} \rho^{a}$ given in (8.5) must be further modified into

$$
\operatorname{Re} \rho^{a}=\hat{a} \mathcal{V}^{a}(v)+h^{a}(v)+\frac{1}{2} \sum_{I} \kappa^{a}\left(Z_{I}, \bar{Z}_{I} ; v\right)+\frac{1}{2 \operatorname{Im} \tau} \mathcal{M}_{\alpha \beta}^{a} \operatorname{Im} \beta^{\alpha} \operatorname{Im} \beta^{\beta}+\frac{1}{2} \mathcal{C}_{\mathcal{I} \mathcal{J}} a^{\mathcal{I}} \bar{a}^{\mathcal{J}}
$$

where

$$
\mathcal{C}_{\mathcal{I} \mathcal{J}}^{a}=-\frac{\mathrm{i}}{2} \int_{\mathcal{C}^{a}} \eta_{\mathcal{I}} \wedge \bar{\eta}_{\mathcal{J}}
$$

with $\mathcal{C}^{a} \equiv D^{a} \cap \Sigma$. The formula (8.10) can be obviously extended to include the contribution of additional D7-branes. Notice that the contribution of the Wilson lines to (8.10) has the same form of the contribution identified in [21] by using probe D7-branes on unwarped compactifications.

\subsection{Complete Kähler potential for $h_{+}^{1,1}=1$}

The new relations (8.10) should be partially inverted in order to find $\hat{a}$ as function of the chiral fields $\rho^{a}, Z_{I}^{i}, \beta^{\alpha}$ and $a^{\mathcal{I}}$, and to properly interpret the Kähler potential as a function of the chiral coordinates. The case $h_{+}^{1,1}=1$ is the simplest one in which one can explicitly invert (8.10). Indeed, proceeding as in section 5.4 one arrives at the Kähler potential

$$
K=-3 \log \left[\operatorname{Re} \rho-\frac{1}{2} \sum_{I} k\left(Z_{I}, \bar{Z}_{I}\right)-\frac{1}{2 \operatorname{Im} \tau} \mathcal{M}_{\alpha \beta} \operatorname{Im} \beta^{\alpha} \operatorname{Im} \beta^{\beta}-\frac{1}{2} \mathcal{C}_{\mathcal{I} \overline{\mathcal{J}}} a^{\mathcal{I}} \bar{a}^{\overline{\mathcal{J}}}\right]+c_{0}+3 \log 3
$$


If we had used in (8.10) the more general universal modulus $a$ instead of $\hat{a}$, we would have obtained the Kähler potential

$K=-3 \log \left[\operatorname{Re} \rho-\frac{1}{2} \sum_{I} k\left(Z_{I}, \bar{Z}_{I}\right)-\frac{1}{2 \operatorname{Im} \tau} \mathcal{M}_{\alpha \beta} \operatorname{Im} \beta^{\alpha} \operatorname{Im} \beta^{\beta}-\frac{1}{2} \mathcal{C}_{\mathcal{I} \overline{\mathcal{J}}} a^{\mathcal{I}} \bar{a}^{\overline{\mathcal{J}}}+3 \mathrm{v}_{0}^{\mathrm{w}}\right]+c_{0}+3 \log 3$

with $\mathrm{v}_{0}^{\mathrm{w}}$ defined in (4.3). Ignoring the contribution of mobile D3-branes and D7-brane Wilson lines, this matches the Kähler potential obtained in [31], using a strategy similar to the one followed in the present paper, which was then confirmed by a direct dimensional reduction in [29].

\subsection{Kähler metric and no-scale structure}

In order to compute the Kähler metric one must use the derivatives of the geometric moduli discussed in appendix A.3. The final result is

$$
\begin{aligned}
\mathcal{L}_{\text {kin }}=-M_{\mathrm{P}}^{2} \mathcal{G}_{a b}^{\mathrm{w}} \nabla_{\mu} \rho^{a} \nabla^{\mu} \bar{\rho}^{b}-\frac{1}{2 \mathrm{v}_{0} \hat{a}} M_{\mathrm{P}}^{2} \sum_{I} g_{0, i \bar{\jmath}}\left(Z_{I}, \bar{Z}_{I}\right) \partial_{\mu} Z_{I}^{i} \partial^{\mu} \bar{Z}_{I}^{\bar{\jmath}} \\
-\frac{1}{4 \mathrm{v}_{0} \hat{a} \operatorname{Im} \tau} M_{\mathrm{P}}^{2} v_{a} \mathcal{M}_{\alpha \beta}^{a} \partial_{\mu} \beta^{\alpha} \partial^{\mu} \bar{\beta}^{\beta}-\frac{1}{2 \mathrm{v}_{0} \hat{a}} M_{\mathrm{P}}^{2} v_{a} \mathcal{C}_{\mathcal{I} \mathcal{J}}^{a} \partial_{\mu} a^{\mathcal{I}} \partial^{\mu} \bar{a}^{\mathcal{J}}
\end{aligned}
$$

where $\mathcal{G}_{a b}^{\mathrm{w}}$ is as in (6.2) and the covariant derivatives are now defined as follows:

$$
\nabla_{\mu} \rho^{a} \equiv \partial_{\mu} \rho^{a}-\mathcal{A}_{i}^{a I} \partial_{\mu} Z_{I}^{i}+\frac{\mathrm{i}}{\operatorname{Im} \tau} \mathcal{M}_{\alpha \beta}^{a} \operatorname{Im} \beta^{\alpha} \partial_{\mu} \beta^{\beta}-\mathcal{C}_{\mathcal{I}}^{a} \bar{a}^{\mathcal{J}} \partial_{\mu} a^{\mathcal{I}}
$$

and $\nabla_{\mu} \bar{\rho}^{a} \equiv\left(\nabla_{\mu} \rho^{a}\right)^{*}$.

By ignoring D3-branes, in the constant warping approximation the kinetic terms (8.14) are consistent with the results of [21]. In particular, as a direct check of the numerical factors, the last term on the r.h.s. of (8.14) can be easily reproduced by dimensionally reducing the DBI action for a probe D7-brane on the metric (3.13).

Furthermore, one can verify that the no-scale condition (6.12) is still satisfied, as required by physical consistency.

Finally, by performing the large moduli expansion discussed in section 7, one can obtain the appropriate modification of (7.4) or, equivalenty, of (7.6). For instance, the latter reads

$$
\begin{aligned}
K \simeq-2 \log \left[\frac{1}{3 !} \mathcal{I}^{a b c} \hat{v}_{a} \hat{v}_{b} \hat{v}_{c}-\frac{1}{2} h(\hat{v})-\frac{1}{4} \sum_{I} k\left(Z_{I}, \bar{Z}_{I} ; \hat{v}\right)\right. \\
\\
\left.\quad-\frac{1}{4 \operatorname{Im} \tau} \hat{v}_{a} \mathcal{M}_{\alpha \beta}^{a} \operatorname{Im} \beta^{\alpha} \operatorname{Im} \beta^{\beta}-\frac{1}{4} \hat{v}_{a} \mathcal{C}_{\mathcal{I} \mathcal{J}}^{a} a^{\mathcal{I}} \overline{\mathcal{J}}\right]-\log \mathrm{v}_{0}+c_{0}
\end{aligned}
$$

where $\hat{v}_{a}$ are functions of $\operatorname{Re} \rho^{a}$ implicitly determined by $\operatorname{Re} \rho^{a}=\frac{1}{2} \mathcal{I}^{a b c} \hat{v}_{b} \hat{v}_{c}$.

\section{Acknowledgments}

I would like to thank S. Andriolo, S. Giusto, E. Plauschinn, R. Savelli, R. Valandro and especially T. Weigand for useful discussions and comments on the draft. The author is 
grateful to the Mainz Institute for Theoretical Physics (MITP) for its hospitality and its partial support during the completion of this work. This work is supported in part by the Padua University Project CPDA144437.

\section{A Derivatives of geometric moduli}

The relations (5.30), (8.5) and (8.10) implicitly define the geometric moduli $\hat{a}$ and $v_{a}$ as functions of the chiral fields $\rho^{a}, Z_{I}^{i}, \beta^{a}$ and $a^{\mathcal{I}}$. Even though in general one cannot make such dependence explicit, it is possible to compute the derivatives of $\hat{a}$ and $v_{a}$ with respect to the chiral fields.

\section{A.1 Useful preliminary formulas}

Once we have chosen the integral two-forms $\omega^{a}$ to be $(1,1)$ and harmonic, we can expand the normalised Kähler form as

$$
J_{0}=v_{a} \omega^{a}
$$

Notice that the $\omega^{a}$ 's, being harmonic, depend on the constrained Kähler moduli $v_{a}$. Now, since any Kähler structure deformation should preserve (2.2), one can easily adapt the discussion for Ricci-flat Calabi-Yau spaces [59] to argue that any infinitesimal Kähler structure deformation must take the form

$$
\delta J_{0}=\delta v_{a} \omega^{a}
$$

By consistency, this implies that

$$
v_{a} \delta \omega^{a}=0
$$

Moreover the normalisation condition (5.3) implies that

$$
\mathcal{I}^{a b c} v_{a} v_{b} \delta v_{b}=0
$$

where $\mathcal{I}^{a b c}$ are the intersection numbers defined in (5.4).

Another useful identity is

$$
\left.\frac{1}{2} J_{0} \wedge J_{0} \wedge \omega^{a}=\frac{1}{3 !}\left(J_{0}\right\lrcorner \omega^{a}\right) J_{0} \wedge J_{0} \wedge J_{0}=d^{a}(v) \operatorname{dvol}_{X, 0}
$$

where

$$
\left.d^{a}(v) \equiv J_{0}\right\lrcorner \omega^{a} \equiv \frac{1}{2 \mathrm{v}_{0}} \mathcal{I}^{a b c} v_{b} v_{c}
$$

The constraint (A.4) implies $\delta v_{a} d^{a}=0$ and then

$$
J_{0} \wedge J_{0} \wedge \delta J_{0}=\delta v_{a} J_{0} \wedge J_{0} \wedge \omega^{a}=0
$$

In other words, the normalisation condition (5.3) requires $\delta J_{0}$ to be primitive.

In the following computations one of the main subtleties comes from the dependence of the potentials $\kappa^{a}(z, \bar{z} ; v)$, defined by $(5.17)$, on the constrained Kähler moduli $v_{a}$. Under a deformation $\delta v_{a}$, we can write

$$
\delta \omega^{a}=\mathrm{i} \partial \bar{\partial} \delta \kappa^{a}
$$


where, since the cohomology class of $\omega^{a}$ is fixed, the variations $\delta \kappa^{a}$ are globally defined functions on $X$. Now, by using (A.8) and (A.2), the constancy of (A.6) implies the following equation for $\delta \kappa^{a}$

$$
\left.\Delta_{0} \delta \kappa^{a}=-2\left(\delta d^{a}+\delta v_{b} \omega^{a}\right\lrcorner \omega^{b}\right)
$$

Consistently, the r.h.s. is a coexact function since from (A.6) one can check that

$$
\left.\delta d^{a}=\frac{1}{\mathrm{v}_{0}} \mathcal{I}^{a b c} v_{b} \delta v_{c}=-\frac{1}{\mathrm{v}_{0}} \delta v_{b} \int_{X} \omega^{a}\right\lrcorner \omega^{b} \mathrm{dvol}_{X, 0}
$$

eq. (A.9) can be integrated, up to an irrelevant constant along $X$, by means of the Green's function $G\left(y, y^{\prime}\right)$ introduced in section 4 :

$$
\delta \kappa^{a}(y ; v)=2 \delta v_{b} \int_{X, y^{\prime}} G\left(y ; y^{\prime}\right)\left(J_{0} \wedge \omega^{a} \wedge \omega^{b}\right)\left(y^{\prime}\right)
$$

Notice that from (A.7) it follows that $v_{a} \delta \kappa^{a}=0$, consistently with (A.3).

Now, recalling the definition $(5.28)$ of $h^{a}(v)$, observe that

$$
\delta h^{a}(v)+\frac{1}{2} \sum_{I} \delta \kappa^{a}\left(Z_{I}, \bar{Z}_{I} ; v\right)=\frac{1}{2 \ell_{\mathrm{s}}^{4}} \int_{X} \delta \kappa^{a} Q_{6}
$$

Combining this equation with (A.11) and (4.6), one can deduce the following crucial formula: ${ }^{12}$

$$
\delta h^{a}(v)+\frac{1}{2} \sum_{I} \delta \kappa^{a}\left(Z_{I}, \bar{Z}_{I} ; v\right)=\delta v_{b} \int_{X} e^{-4 \hat{A}_{0}} J_{0} \wedge \omega^{a} \wedge \omega^{b}
$$

\section{A.2 Derivatives in absence of axionic chiral fields}

We are now ready to compute the derivatives of the background geometric moduli with respect to the chiral fields. Take first the derivatives of (5.30) with respect to $\operatorname{Re} \rho^{b}$. By using (A.13) one gets

$$
\delta_{b}^{a}=\frac{\partial \hat{a}}{\partial \operatorname{Re} \rho^{b}} \mathcal{V}^{a}+\mathcal{I}_{\mathrm{w}}^{a c d} v_{c} \frac{\partial v_{d}}{\partial \operatorname{Re} \rho^{b}}
$$

where we have introduced the warped 'intersection numbers'

$$
\mathcal{I}_{\mathrm{w}}^{a b c} \equiv \int_{X} e^{-4 A} \omega^{a} \wedge \omega^{b} \wedge \omega^{c}
$$

Notice that $\mathcal{I}_{\mathrm{w}}^{a b c}$ is not a topological quantity and that by (A.7) we have

$$
\mathcal{I}_{\mathrm{w}}^{a b c} v_{a} v_{b} \delta v_{b}=0
$$

Let us also define

$$
M_{\mathrm{w}}^{a b} \equiv \int_{X} e^{-4 A} J_{0} \wedge \omega^{a} \wedge \omega^{b} \equiv \mathcal{I}_{\mathrm{w}}^{a b c} v_{c}
$$

\footnotetext{
${ }^{12}$ In deducing the formula (A.13) we have neglected the possible dependence on the Kähler moduli of the higher-order curvature corrections appearing in $Q_{6}$. Taking such dependence into account would require to work beyond the second derivative order in the effective action.
} 
Then from (A.14) one can derive

$$
\begin{aligned}
\frac{\partial \hat{a}}{\partial \operatorname{Re} \rho^{a}} & =\frac{v_{a}}{3 \mathrm{v}_{0}} \\
\frac{\partial v_{a}}{\partial \operatorname{Re} \rho^{b}} & =\left(M_{\mathrm{w}}^{-1}\right)_{a b}-\frac{1}{6 \mathrm{v}_{0} \hat{a}} v_{a} v_{b}
\end{aligned}
$$

Indeed, (A.18a) can be obtained by contracting (A.14) with $v_{a}$ and using (A.16). Then, one can determine (A.18b) by substituting (A.18a) in (A.14) and by observing that

$$
M_{\mathrm{w}}^{a b} v_{b}=2 \hat{a} \mathcal{V}^{a}(v)
$$

which follows from (A.5) and (4.7).

Starting back from (5.30), taking its derivative with respect to $Z_{I}^{i}$ and using again (A.13) one gets

$$
0=\frac{\partial \hat{a}}{\partial Z_{I}^{i}} \mathcal{V}^{a}+M_{\mathrm{w}}^{a b} \frac{\partial v_{b}}{\partial Z_{I}^{i}}+\frac{1}{2} \frac{\partial \kappa^{a}\left(Z_{I}, \bar{Z}_{I} ; v\right)}{\partial Z_{I}^{i}}
$$

Then, proceeding as in the previous case, we arrive at

$$
\begin{aligned}
\frac{\partial \hat{a}}{\partial Z_{I}^{i}} & =-\frac{v_{a}}{6 \mathrm{v}_{0}} \frac{\partial \kappa^{a}\left(Z_{I}, \bar{Z}_{I} ; v\right)}{\partial Z_{I}^{i}} \\
\frac{\partial v_{a}}{\partial Z_{I}^{i}} & =\frac{1}{2}\left[\frac{1}{6 \mathrm{v}_{0} \hat{a}} v_{a} v_{b}-\left(M_{\mathrm{w}}^{-1}\right)_{a b}\right] \frac{\partial \kappa^{b}\left(Z_{I}, \bar{Z}_{I} ; v\right)}{\partial Z_{I}^{i}}
\end{aligned}
$$

\section{A.3 Derivatives in presence of axionic chiral fields}

The discussion of the previous subsection can be repeated starting from (8.5) or more generically (8.10). Let collectively denote the additional chiral fields $\beta^{a}$ and $a^{\mathcal{I}}$ with $\phi^{\mathbb{A}}$ and rewrite (8.5) or (8.10) in the general form

$$
\operatorname{Re} \rho^{a}=\hat{a} \mathcal{V}^{a}(v)+\frac{1}{2} \sum_{I} \kappa^{a}\left(Z_{I}, \bar{Z}_{I} ; v\right)+h^{a}(v)+N^{a}(\phi, \bar{\phi})
$$

where $N^{a}(\phi, \bar{\phi})$ does not depend on the chiral fields $\rho^{a}$ and $Z_{I}^{i}$. Proceeding as in the previous subsection one again arrives at (A.18) and (A.21). In addition, one also gets

$$
\begin{aligned}
\frac{\partial \hat{a}}{\partial \phi^{\mathbb{A}}} & =-\frac{1}{3 \mathrm{v}_{0}} v_{a} N_{\mathbb{A}}^{a} \\
\frac{\partial v_{a}}{\partial \phi^{\mathbb{A}}} & =\left[\frac{1}{6 \mathrm{v}_{0} \hat{a}} v_{a} v_{b}-\left(M_{\mathrm{w}}^{-1}\right)_{a b}\right] N_{\mathbb{A}}^{b}
\end{aligned}
$$

where $N_{\mathbb{A}} \equiv \frac{\partial N^{a}}{\partial \phi^{\mathbb{A}}}$.

\section{B A simple example}

In this appendix we apply our results to a simple concrete class of $N=1$ compactifications on $X=T^{6} / \mathbb{Z}_{2}$ [35], for which the Kähler potential can be made fully explicit. The internal 
six-torus is factorised, $T^{6}=T^{2} \times T^{2} \times T^{2}$, and the corresponding complex coordinates $\left(z^{1}, z^{2}, z^{3}\right)$ are periodically identified according to the rule $z^{i} \simeq z^{i}+n^{i}+\lambda m^{i}$ (no sum over $i$ ), where $n^{i}, m^{i} \in \mathbb{Z}$ and $\lambda \equiv e^{\frac{2 \pi \mathrm{i}}{3}}$ defines the complex structure modulus of the three tori. The axio-dilaton takes the value $\tau=e^{\frac{2 \pi \mathrm{i}}{3}}$ too. The $\mathbb{Z}_{2}$ orientifold involution is defined by $z^{i} \rightarrow-z^{i}$. One can choose a supersymmetric $G_{3}$ flux of the form

$$
G_{3} \sim \mathrm{d} z^{1} \wedge \mathrm{d} z^{2} \wedge \mathrm{d} \bar{z}^{3}+\mathrm{d} z^{2} \wedge \mathrm{d} z^{3} \wedge \mathrm{d} \bar{z}^{1}+\mathrm{d} z^{3} \wedge \mathrm{d} z^{1} \wedge \mathrm{d} \bar{z}^{1}
$$

The flux quantisation condition fixes the number of D3-branes. In [35] one can find an example with $N_{\mathrm{D} 3}=10$.

On $T^{6} / \mathbb{Z}_{2}$ one has $h_{+}^{1,1}=9$, but the primitivity requirement $J_{0} \wedge G_{3}=0$ imposes additional conditions. Such conditions are not explicitly taken into account in the rest of the present paper but, as this appendix shows, they can be easily incorporated.

Even though one would generically expect six non-trivial conditions from $J_{0} \wedge G_{3}=0$, the simplicity of (B.1) implies that only three conditions are effective. These select six out of the nine independent integral even $(1,1)$ forms, which can be chosen as follows:

$$
\begin{array}{llrl}
\omega^{1} & =\frac{\mathrm{i}}{\operatorname{Im} \lambda} \mathrm{d} z^{1} \wedge \mathrm{d} \bar{z}^{1}, & \omega^{2}=\frac{\mathrm{i}}{\operatorname{Im} \lambda} \mathrm{d} z^{2} \wedge \mathrm{d} \bar{z}^{2}, & \omega^{3}=\frac{\mathrm{i}}{\operatorname{Im} \lambda} \mathrm{d} z^{3} \wedge \mathrm{d} \bar{z}^{3}, \\
\omega^{4}=\frac{1}{\operatorname{Im} \lambda} \operatorname{Im}\left(\mathrm{d} \bar{z}^{2} \wedge \mathrm{d} z^{3}\right), & \omega^{5}=\frac{1}{\operatorname{Im} \lambda} \operatorname{Im}\left(\mathrm{d} \bar{z}^{3} \wedge \mathrm{d} z^{1}\right), & \omega^{6}=\frac{1}{\operatorname{Im} \lambda} \operatorname{Im}\left(\mathrm{d} \bar{z}^{1} \wedge \mathrm{d} z^{2}\right)
\end{array}
$$

The non-vanishing intersection numbers $\mathcal{I}^{a b c}=\frac{1}{2} \int_{T^{6}} \omega^{a} \wedge \omega^{b} \wedge \omega^{c}$ are given by

$$
\mathcal{I}^{123}=4, \quad \mathcal{I}^{456}=1, \quad \mathcal{I}^{144}=\mathcal{I}^{255}=\mathcal{I}^{366}=-2
$$

Then the constraint (5.3) takes the form

$$
4 v_{1} v_{2} v_{3}+v_{4} v_{5} v_{6}-v_{1} v_{4}^{2}-v_{2} v_{5}^{2}-v_{3} v_{6}^{2}=\mathrm{v}_{0}
$$

Since the Kähler form $J_{0}=v_{a} \omega^{a}$ as well as the two-forms $\omega^{a}$ have constant coefficients, $\omega^{a}$ are automatically harmonic. In particular they do not depend on the constrained Kähler moduli and the potentials

$$
\begin{array}{lll}
\kappa^{1}=\frac{z^{1} \bar{z}^{1}}{\operatorname{Im} \lambda} & \kappa^{2}=\frac{z^{2} \bar{z}^{2}}{\operatorname{Im} \lambda} & \kappa^{3}=\frac{z^{3} \bar{z}^{3}}{\operatorname{Im} \lambda} \\
\kappa^{4}=\frac{\operatorname{Re}\left(z^{2} \bar{z}^{3}\right)}{\operatorname{Im} \lambda} & \kappa^{5}=\frac{\operatorname{Re}\left(z^{3} \bar{z}^{1}\right)}{\operatorname{Im} \lambda} & \kappa^{6}=\frac{\operatorname{Re}\left(z^{1} \bar{z}^{2}\right)}{\operatorname{Im} \lambda}
\end{array}
$$

do not depend on the moduli $v_{a}$ either. This implies the drastic simplification that the functions $h^{a}(v)$ defined in (5.28) are constant, so that they can be ignored in (5.30). 
Hence, the geometric moduli $\hat{a}$ and $v_{a}$ are related to the chiral fields $\rho^{a}$ and $Z_{I}^{i}$ by

$$
\begin{gathered}
\hat{a}\left(4 v_{2} v_{3}-v_{4}^{2}\right)=\operatorname{Re} \rho^{1}-\frac{1}{2 \operatorname{Im} \lambda} \sum_{I} Z_{I}^{1} \bar{Z}_{I}^{1} \equiv T^{1} \\
\hat{a}\left(4 v_{1} v_{3}-v_{5}^{2}\right)=\operatorname{Re} \rho^{2}-\frac{1}{2 \operatorname{Im} \lambda} \sum_{I} Z_{I}^{2} \bar{Z}_{I}^{2} \equiv T^{2} \\
\hat{a}\left(4 v_{1} v_{2}-v_{6}^{2}\right)=\operatorname{Re} \rho^{3}-\frac{1}{2 \operatorname{Im} \lambda} \sum_{I} Z_{I}^{3} \bar{Z}_{I}^{3} \equiv T^{3} \\
\hat{a}\left(v_{5} v_{6}-2 v_{1} v_{4}\right)=\operatorname{Re} \rho^{4}-\frac{1}{2 \operatorname{Im} \lambda} \sum_{I} \operatorname{Re}\left(Z_{I}^{2} \bar{Z}_{I}^{3}\right) \equiv T^{4} \\
\hat{a}\left(v_{4} v_{6}-2 v_{2} v_{5}\right)=\operatorname{Re} \rho^{5}-\frac{1}{2 \operatorname{Im} \lambda} \sum_{I} \operatorname{Re}\left(Z_{I}^{3} \bar{Z}_{I}^{1}\right) \equiv T^{5} \\
\hat{a}\left(v_{4} v_{5}-2 v_{3} v_{6}\right)=\operatorname{Re} \rho^{6}-\frac{1}{2 \operatorname{Im} \lambda} \sum_{I} \operatorname{Re}\left(Z_{I}^{1} \bar{Z}_{I}^{2}\right) \equiv T^{6}
\end{gathered}
$$

where we have introduced the set of real functions

$$
T^{a}(\operatorname{Re} \rho, Z, \bar{Z}) \equiv \operatorname{Re} \rho^{a}-\frac{1}{2} \sum_{I} \kappa^{a}\left(Z_{I}, \bar{Z}_{I}\right)
$$

By using (B.4) one can partially invert the relations (B.6) to find the function $\hat{a}(\operatorname{Re} \rho, Z, \bar{Z})$ and then write the Kähler potential (5.32) as follows:

$$
K=-\log \left[T^{1} T^{2} T^{3}+2 T^{4} T^{5} T^{6}-T^{1}\left(T^{4}\right)^{2}-T^{2}\left(T^{5}\right)^{2}-T^{3}\left(T^{6}\right)^{2}\right]-\log \frac{\mathrm{v}_{0}}{4}+c_{0}
$$

So for these simple models one can find a fully explicit Kähler potential. On the other hand, these models are too simple to exhibit non-trivial warping effects due to the fluxes.

Open Access. This article is distributed under the terms of the Creative Commons Attribution License (CC-BY 4.0), which permits any use, distribution and reproduction in any medium, provided the original author(s) and source are credited.

\section{References}

[1] K. Becker and M. Becker, M theory on eight manifolds, Nucl. Phys. B 477 (1996) 155 [hep-th/9605053] [INSPIRE].

[2] K. Dasgupta, G. Rajesh and S. Sethi, $M$ theory, orientifolds and G-flux, JHEP 08 (1999) 023 [hep-th/9908088] [INSPIRE].

[3] M. Graña and J. Polchinski, Supersymmetric three form flux perturbations on $A d S_{5}$, Phys. Rev. D 63 (2001) 026001 [hep-th/0009211] [INSPIRE].

[4] S.S. Gubser, Supersymmetry and F-theory realization of the deformed conifold with three form flux, hep-th/0010010 [INSPIRE].

[5] S.B. Giddings, S. Kachru and J. Polchinski, Hierarchies from fluxes in string compactifications, Phys. Rev. D 66 (2002) 106006 [hep-th/0105097] [INSPIRE]. 
[6] M. Graña and J. Polchinski, Gauge/gravity duals with holomorphic dilaton, Phys. Rev. D 65 (2002) 126005 [hep-th/0106014] [INSPIRE].

[7] K. Becker and M. Becker, Supersymmetry breaking, M-theory and fluxes, JHEP 07 (2001) 038 [hep-th/0107044] [INSPIRE].

[8] F. Denef, Les Houches lectures on constructing string vacua, arXiv:0803.1194 [INSPIRE].

[9] A. Maharana and E. Palti, Models of particle physics from type IIB string theory and F-theory: a review, Int. J. Mod. Phys. A 28 (2013) 1330005 [arXiv:1212.0555] [InSPIRE].

[10] O. DeWolfe and S.B. Giddings, Scales and hierarchies in warped compactifications and brane worlds, Phys. Rev. D 67 (2003) 066008 [hep-th/0208123] [INSPIRE].

[11] S. Kachru, R. Kallosh, A.D. Linde and S.P. Trivedi, De Sitter vacua in string theory, Phys. Rev. D 68 (2003) 046005 [hep-th/0301240] [INSPIRE].

[12] S. Kachru et al., Towards inflation in string theory, JCAP 10 (2003) 013 [hep-th/0308055] [INSPIRE].

[13] S.B. Giddings and A. Maharana, Dynamics of warped compactifications and the shape of the warped landscape, Phys. Rev. D 73 (2006) 126003 [hep-th/0507158] [INSPIRE].

[14] A.R. Frey and A. Maharana, Warped spectroscopy: localization of frozen bulk modes, JHEP 08 (2006) 021 [hep-th/0603233] [INSPIRE].

[15] C.P. Burgess et al., Warped supersymmetry breaking, JHEP 04 (2008) 053 [hep-th/0610255] [INSPIRE].

[16] F. Marchesano, P. McGuirk and G. Shiu, Open string wavefunctions in warped compactifications, JHEP 04 (2009) 095 [arXiv: 0812.2247] [INSPIRE].

[17] F. Marchesano, P. McGuirk and G. Shiu, Chiral matter wavefunctions in warped compactifications, JHEP 05 (2011) 090 [arXiv: 1012.2759] [INSPIRE].

[18] T.W. Grimm, D. Klevers and M. Poretschkin, Fluxes and warping for gauge couplings in F-theory, JHEP 01 (2013) 023 [arXiv: 1202.0285] [INSPIRE].

[19] M. Graña, T.W. Grimm, H. Jockers and J. Louis, Soft supersymmetry breaking in Calabi-Yau orientifolds with D-branes and fluxes, Nucl. Phys. B 690 (2004) 21 [hep-th/0312232] [INSPIRE].

[20] T.W. Grimm and J. Louis, The effective action of $N=1$ Calabi-Yau orientifolds, Nucl. Phys. B 699 (2004) 387 [hep-th/0403067] [INSPIRE].

[21] H. Jockers and J. Louis, The effective action of D7-branes in $N=1$ Calabi-Yau orientifolds, Nucl. Phys. B 705 (2005) 167 [hep-th/0409098] [INSPIRE].

[22] H. Jockers and J. Louis, D-terms and F-terms from D7-brane fluxes, Nucl. Phys. B 718 (2005) 203 [hep-th/0502059] [INSPIRE].

[23] T.W. Grimm, The $N=1$ effective action of F-theory compactifications, Nucl. Phys. B 845 (2011) 48 [arXiv: 1008.4133] [InSPIRE].

[24] G. Shiu, G. Torroba, B. Underwood and M.R. Douglas, Dynamics of warped flux compactifications, JHEP 06 (2008) 024 [arXiv:0803.3068] [INSPIRE].

[25] M.R. Douglas and G. Torroba, Kinetic terms in warped compactifications, JHEP 05 (2009) 013 [arXiv: 0805.3700] [inSPIRE]. 
[26] A.R. Frey, G. Torroba, B. Underwood and M.R. Douglas, The universal Kähler modulus in warped compactifications, JHEP 01 (2009) 036 [arXiv:0810.5768] [INSPIRE].

[27] H.-Y. Chen, Y. Nakayama and G. Shiu, On D3-brane dynamics at strong warping, Int. J. Mod. Phys. A 25 (2010) 2493 [arXiv:0905.4463] [InSPIRE].

[28] B. Underwood, A breathing mode for warped compactifications, Class. Quant. Grav. 28 (2011) 195013 [arXiv:1009.4200] [INSPIRE].

[29] A.R. Frey and J. Roberts, The dimensional reduction and Kähler metric of forms in flux and warping, JHEP 10 (2013) 021 [arXiv:1308.0323] [INSPIRE].

[30] P. Koerber and L. Martucci, From ten to four and back again: How to generalize the geometry, JHEP 08 (2007) 059 [arXiv:0707.1038] [INSPIRE].

[31] L. Martucci, On moduli and effective theory of $N=1$ warped flux compactifications, JHEP 05 (2009) 027 [arXiv:0902.4031] [INSPIRE].

[32] E. Cremmer, S. Ferrara, C. Kounnas and D.V. Nanopoulos, Naturally vanishing cosmological constant in $N=1$ supergravity, Phys. Lett. B 133 (1983) 61 [InSPIRE].

[33] J.R. Ellis, A.B. Lahanas, D.V. Nanopoulos and K. Tamvakis, No-scale supersymmetric standard model, Phys. Lett. B 134 (1984) 429 [INSPIRE].

[34] D. Baumann et al., On D3-brane potentials in compactifications with fluxes and wrapped D-branes, JHEP 11 (2006) 031 [hep-th/0607050] [INSPIRE].

[35] S. Kachru, M.B. Schulz and S. Trivedi, Moduli stabilization from fluxes in a simple IIB orientifold, JHEP 10 (2003) 007 [hep-th/0201028] [INSPIRE].

[36] M. Haack and J. Louis, Duality in heterotic vacua with four supercharges, Nucl. Phys. B 575 (2000) 107 [hep-th/9912181] [INSPIRE].

[37] M. Haack and J. Louis, M theory compactified on Calabi-Yau fourfolds with background flux, Phys. Lett. B 507 (2001) 296 [hep-th/0103068] [INSPIRE].

[38] T.W. Grimm, J. Keitel, R. Savelli and M. Weissenbacher, From M-theory higher curvature terms to $\alpha^{\prime}$ corrections in F-theory, arXiv:1312.1376 [INSPIRE].

[39] D. Junghans and G. Shiu, Brane curvature corrections to the $\backslash=1$ type II/F-theory effective action, arXiv: 1407.0019 [INSPIRE].

[40] J. Gray and A. Lukas, Gauge five-brane moduli in four-dimensional heterotic models, Phys. Rev. D 70 (2004) 086003 [hep-th/0309096] [INSPIRE].

[41] R. Kallosh, L. Kofman, A.D. Linde and A. Van Proeyen, Superconformal symmetry, supergravity and cosmology, Class. Quant. Grav. 17 (2000) 4269 [Erratum ibid. 21 (2004) 5017] [hep-th/0006179] [INSPIRE].

[42] S. Gukov, C. Vafa and E. Witten, CFT's from Calabi-Yau four folds, Nucl. Phys. B 584 (2000) 69 [Erratum ibid. B 608 (2001) 477-478] [hep-th/9906070] [INSPIRE].

[43] M. Graña, R. Minasian, M. Petrini and A. Tomasiello, Generalized structures of $N=1$ vacua, JHEP 11 (2005) 020 [hep-th/0505212] [INSPIRE].

[44] P. Warner, Foundations of differentiable manifolds and Lie groups, Scott, Foresman and Co., U.S.A. (1971). 
[45] E. Witten, World sheet corrections via D instantons, JHEP 02 (2000) 030 [hep-th/9907041] [INSPIRE].

[46] L. Martucci and P. Smyth, Supersymmetric D-branes and calibrations on general $N=1$ backgrounds, JHEP 11 (2005) 048 [hep-th/0507099] [INSPIRE].

[47] M. Bianchi, A. Collinucci and L. Martucci, Magnetized E3-brane instantons in F-theory, JHEP 12 (2011) 045 [arXiv:1107.3732] [INSPIRE].

[48] M. Bianchi, G. Inverso and L. Martucci, Brane instantons and fluxes in F-theory, JHEP 07 (2013) 037 [arXiv: 1212.0024] [INSPIRE].

[49] L. Martucci, Topological duality twist and brane instantons in F-theory, JHEP 06 (2014) 180 [arXiv: 1403.2530] [INSPIRE].

[50] P. Griffiths and J. Harris, Principles of algebraic geometry, Wiley-Interscience Publications, U.S.A. (1994).

[51] O.J. Ganor, A note on zeros of superpotentials in F-theory, Nucl. Phys. B 499 (1997) 55 [hep-th/9612077] [INSPIRE].

[52] A. Dymarsky and L. Martucci, D-brane non-perturbative effects and geometric deformations, JHEP 04 (2011) 061 [arXiv: 1012.4018] [INSPIRE].

[53] F. Marchesano and L. Martucci, Non-perturbative effects on seven-brane Yukawa couplings, Phys. Rev. Lett. 104 (2010) 231601 [arXiv:0910.5496] [INSPIRE].

[54] S. Ferrara, L. Girardello, T. Kugo and A. Van Proeyen, Relation between different auxiliary field formulations of $N=1$ supergravity coupled to matter, Nucl. Phys. B 223 (1983) 191 [INSPIRE].

[55] S. Cecotti, S. Ferrara and M. Villasante, Linear multiplets and super Chern-Simons forms in $4 D$ supergravity, Int. J. Mod. Phys. A 2 (1987) 1839 [inSPIRE].

[56] D. Kotschik, On products of harmonic forms, Duke Math. J. 107 (2001) 521 [math/0004009].

[57] P. Pasti, D.P. Sorokin and M. Tonin, Covariant action for a $D=11$ five-brane with the chiral field, Phys. Lett. B 398 (1997) 41 [hep-th/9701037] [INSPIRE].

[58] I.A. Bandos et al., Covariant action for the superfive-brane of M-theory, Phys. Rev. Lett. 78 (1997) 4332 [hep-th/9701149] [INSPIRE].

[59] P. Candelas and X. de la Ossa, Moduli Space of Calabi-Yau Manifolds, Nucl. Phys. B 355 (1991) 455 [inSPIRE]. 